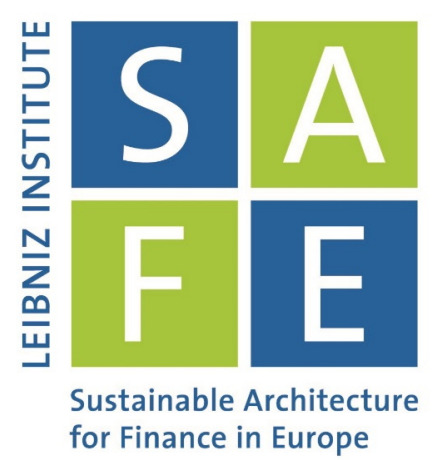

Andreas Hackethal | Michael Kirchler | Christine Laudenbach | Michael Razen | Annika Weber

\title{
On the (Ir)Relevance of Monetary Incentives in Risk Preference Elicitation Experiments
}

SAFE Working Paper No. 286

\section{Leibniz Institute for Financial Research SAFE} Sustainable Architecture for Finance in Europe 


\title{
On the (ir)relevance of monetary incentives in risk preference elicitation experiments*
}

\author{
Andreas Hackethal ${ }^{\dagger} \quad$ Michael Kirchler ${ }^{\ddagger} \quad$ Christine Laudenbach $^{\S}$ \\ Michael Razen ${ }^{\ddagger} \quad$ Annika Weber $^{\dagger}$
}

August 31, 2020

\begin{abstract}
Incentivized experiments in which individuals receive monetary rewards according to the outcomes of their decisions are regarded as the gold standard for preference elicitation in experimental economics. These task-related real payments are considered necessary to reveal subjects' "true preferences". Using a systematic, large-sample approach with three subject pools of private investors, professional investors, and students, we test the effect of task-related monetary incentives on risk preferences elicited in four standard experimental tasks. We find no systematic differences in behavior between subjects in the incentivized and non-incentivized regimes. We discuss implications for academic research and for applications in the field.
\end{abstract}

JEL: C91, D01, D81

Keywords: Risk Preferences, Incentives, Experimental Economics, Risk Aversion.

\footnotetext{
${ }^{*}$ We are grateful for helpful comments by Thomas Dohmen, Johannes Hermle, Felix Holzmeister, Alex Imas, Matthias Stefan, and Ruediger Weber on earlier versions of this paper. Financial support from the Austrian Science Fund FWF (START-grant Y617-G11 Kirchler, and SFB F63) is gratefully acknowledged. We gratefully acknowledge research support from the Leibniz Institute for Financial Research SAFE. There exist no conflicts of interest of any of the authors involved in this project. The study was pre-registered, and the data and replication materials will be made public upon publication of the paper. This project was ethically approved by the IRB at Goethe University Frankfurt.

${ }^{\dagger}$ Goethe University Frankfurt and Leibniz Institute for Financial Research SAFE, Frankfurt

${ }^{\ddagger}$ University of Innsbruck

${ }^{\S}$ Goethe University Frankfurt
} 
Risk is inherent to economic decision-making across many real-life domains, such as investments, health behaviors, or labor supply. As risk preferences are a fundamental determinant of decisions under risk, understanding how individuals' preferences feed into decisions is essential to the study of individual decision-making. As a result, assumptions about individuals' attitudes toward risk are central ingredients in many seminal models in economics and finance (e.g. Markowitz, 1952; Merton, 1969; Pratt, 1964; Barberis et al., 2001; Kahneman and Tversky, 1979). Researchers in the decision sciences, such as economics, finance, and neuroscience, commonly use controlled experiments to assess individuals' willingness to take risks. Among these important tools are incentivized experiments, in which individuals receive real monetary rewards according to the outcomes of their decisions. For incentivized experiments, which are considered the gold standard for eliciting individuals' risk preferences, the underlying assumption is that individuals reveal their true preferences only if the experimental tasks have salient monetary consequences (Smith, 1976; Harrison, 1994). This practice of using incentives contrasts with practices in other social sciences, most prominently psychology, where non-incentivized, hypothetical choices are common (Camerer and Hogarth, 1999; Hertwig and Ortmann, 2001).

The strong mandate to incentivize preference-elicitation tasks according to individuals' choices in economic experiments not only induces substantial monetary costs - effectively limiting sample sizes - but also increases administrative efforts (Dohmen et al., 2011). Moreover, complicated payoff formulas may unduly increase the complexity of the experimental design and arguably make choices less realistic to subjects (Read, 2005; Bardsley et al., 2020).

Additionally, the rise of online surveys has facilitated the recruitment of subjects on a large scale and opened the possibility of studying choices and preferences among subject pools other than students. Obtaining accurate measures of risk attitudes of non-standard subjects such as private and professional investors is essential to understand their financial behaviors and to gauge their impact on asset prices and the macroeconomy (Guiso and Sodini, 2013). However, using task-related incentives may not always be feasible in these settings.

Against this background, we use a systematic, large-scale approach to study the impact of task-related monetary incentives on experimentally elicited risk-preference measures. Specifically, we innovate along two dimensions: (i) we consider four standard experimental tasks instead of focusing on a single task, and (ii) we study the choices of private and professional investors in addition to the choices of students. We administer an online experiment to a total sample of 1,727 participants, among them 838 private investors at a large German bank, 251 professional investors at various financial companies in the EU, and 638 students at the University of Innsbruck. The experimental tasks we consider are the staircase procedure by Falk et al. (2016, 2018), the gamble-choice task by Eckel and Grossman (2002), the paired lottery 
choice task by Holt and Laury (2002), and the investment game by Gneezy and Potters (1997). We randomly assign subjects to two incentive conditions. Respondents in the FLAT condition receive a fixed fee as a reward for participation. Respondents in the INCENTIVES condition - in addition to the fixed participation fee - receive a task-related payment according to the outcome of their decision in one randomly selected experimental task.

In 10 of the 12 in-sample comparisons, we find no systematic differences between the participants' choices in the FlAT and the InCEnTIVES condition. However, for the Holt and Laury (2002) elicitation task, we document a small increase in risk aversion for students and professional investors in the INCENTIVES condition, relative to the FLAT condition. We find no systematic differences by incentive condition with respect to other aspects of decision quality, such as effort provided (i.e., task-specific response times, drop-out rates, instances of inconsistent answers) and within-subject consistency in choices across tasks.

Our results complement prior research investigating hypothetical bias in decisions under risk. Studies of whether and how task-related incentives affect subjects' risk-taking in economic experiments have produced mixed results. While some investigations find that subjects' behavior is more risk averse when choices have real consequences (Holt and Laury, 2002, 2005; Harrison et al., 2005) ${ }^{1}$, still other studies find no differences in subjects' choices across incentive conditions (Beattie and Loomes, 1997; Kuehberger et al., 2002). ${ }^{2}$ Gneezy et al. (2015) jointly elicit risk and ambiguity attitudes and find no difference in estimated parameters when incentives are real or hypothetical. Comparing real and hypothetical decisions, Camerer and Mobbs (2017) observe differences in brain activity, although not in all of the decision domains studied. ${ }^{3}$ More recently, Etchart-Vincent and l'Haridon (2011) document that differences in the incentive scheme have no effect in the loss domain, whereas incentives matter for risk-taking in the gain domain. We contribute to this literature by providing a comprehensive picture of the role of incentives in the experimental elicitation of risk preferences. Rather than focusing on a single experimental task, we run a battery of commonly used risk-elicitation experiments. Importantly, with more than 1,700 respondents, our study is high-powered and allows for conclusive inferences even in the case of null results. While we cannot make statements about whether our findings hold with high stake sizes as well, we consider our focus on low and moderate stake sizes to be an advantage, as these are a common

\footnotetext{
${ }^{1}$ Smith and Walker (1993), other research documents that incentives lessen the variance of experimental measures by reducing instances of extreme outliers, potentially caused by inattentive or unmotivated subjects. See Camerer and Hogarth (1999) for a discussion.

${ }^{2}$ For extensive reviews, see Camerer (1995); Camerer and Hogarth (1999); Camerer and Mobbs (2017); Harrison (2006).

${ }^{3}$ Closely related, Dohmen et al. (2011) validate a non-incentivized survey item designed to measure individuals' risk attitudes using a lottery-choice experiment and find that the general risk-attitude question strongly predicts behavior in the experiment. The survey item is also found to generate the best predictor of real-world risky behaviors.
} 
standard in the literature and increase the generalizability of our study for state-of-the-art procedures.

Second, we add to the literature on the generalizability of findings obtained in laboratory experiments with standard student subjects (e.g., List, 2003; Haigh and List, 2005; Alevy et al., 2007). Besides examining the role of incentives in experiments with students as subjects, we run our experiments with two large, non-standard subject pools of private and professional investors. Given the ramifications for asset prices and the macroeconomy, obtaining valid measures of these subjects' risk preferences - and the interplay with task-related incentives is of great interest to academics, regulators, and policy makers.

\section{Experimental Design}

\section{Experimental tasks}

We consider four of the most widely used experimental tasks for eliciting risk preferences, which we introduce below. Table 1 provides details on the parameterization of the gambles involved in each of the tasks. The experimental instructions are provided in Online Appendix D. The euro amounts stated refer to the payoff parameters in the private and professional investor sample. To align stake sizes for all subject pools with the standards in the experimental economics literature, we divide these euro amounts by four to obtain potential payoffs for the student sample (see, e.g., Haigh and List, 2005; Kirchler et al., 2018).

(i) The staircase procedure by Falk et al. $(2016,2018)$ (FA) aims to elicit subjects' certainty equivalent for a given lottery in a series of decisions. We ask subjects to choose between a lottery paying $€ 45$ or $€ 0$ with equal probability, and a safe payment of $€ 24$. Subjects who prefer the lottery in the first stage are offered a higher safe payment $(€ 36)$ in the second decision, whereas subjects who prefer the safe payment are presented a lower safe payment $(€ 12)$. The payoffs of the lottery remain constant across the decision rounds. In our specification, the payout of the safe alternative varies from $€ 3$ to $€ 45$. After four decision rounds, the staircase design allows the researcher to pin down a narrow interval for subjects' certainty equivalent as a measure of their risk preference, with certainty equivalents being higher for more risk-tolerant individuals. We provide an exposition of the entire sequence of decisions in the four decision rounds in Figure A1.

(ii) The gamble-choice task by Eckel and Grossman (2002) (EG) asks subjects to choose their preferred lottery specification from a menu of six 50/50 gambles (see Table 1). The first lottery offers a secure payoff of $€ 21$ in both states of the world. Subsequently, the difference between the two possible payoffs widens, as the first payoff increases by 
$€ 6$ while the second payoff decreases by $€ 3$ in each subsequent lottery. Consequently, the rank of the lottery chosen, ranging from 1 to 6 , serves as a measure of a subject's risk tolerance. Subjects with higher risk tolerance will choose lotteries farther down the list, as these offer higher expected returns at higher levels of risk.

(iii) The paired lottery choice task by Holt and Laury (2002) (HL) presents subjects with 10 separate decisions between a lottery A that pays either $€ 24.00$ or $€ 19.20$ and a lottery B that pays either $€ 46.20$ or $€ 1.20$ (see Table 1 ). In the first decision, the probability for the high [low] state is $10 \%$ [90\%] in both lotteries. In each subsequent decision, the probability of the high [low] state increases [decreases] by $10 \%$. Hence, in each decision, choosing lottery A is less risky than choosing lottery B. At the same time, however, the expected value of lottery A increases from $€ 19.68$ to $€ 24.00$, while the expected value of lottery B increases from $€ 5.70$ to $€ 46.20$. As a measure of individuals' willingness to incur risk, we mark the decision where a subject switches from lottery A to lottery B. Subjects with higher risk tolerance will switch earlier to lottery $\mathrm{B}^{4}{ }^{4}$ For ease of interpretation, we count the number of rows after the switching point, such that higher values imply higher risk tolerance. ${ }^{5}$

(iv) The investment game by Gneezy and Potters (1997) (GP) stylizes an investment decision. In this task, subjects receive an initial endowment of $€ 24$ and are asked to decide which fraction to invest in a project that pays either 2.5 times the invested amount or $€ 0$, with equal probability (see Table 1 ). ${ }^{6}$ The amount not invested is kept in either state of the world. As is apparent from the parametrization, higher investments increase both expected value and variance of the payoff. We use the amount a subject invests in the risky project as a measure of risk tolerance such that higher values indicate higher levels of risk tolerance. Risk-neutral and risk-seeking subjects will invest their entire endowment.

\footnotetext{
${ }^{4}$ Note that in the $10^{\text {th }}$ decision, the higher payoff will be paid with certainty in both lotteries, so choosing the second lottery is a dominant strategy. This result provides validation of whether subjects have understood the task (Charness et al., 2020).

${ }^{5}$ Note that we allow participants to freely switch between options A and B as they move down the 10 decision rows. This lack of constraint allows for the possibility that individuals may behave inconsistently by choosing to switch between lotteries A and B more than once. We drop these observations from our main analyses but include a specific discussion on a potential association between incentives and the propensity to behave inconsistently in section 2 .

${ }^{6}$ We we use the same variant as Charness et al. (2020). Note that in the original version of Gneezy and Potters (1997), the probabilities for receiving 2.5 times the invested amount and zero are $1 / 3$ and $2 / 3$, respectively.
} 
Table 1: Overview of risky choices in the single tasks

\section{Task Choice(s)}

FA Sequence of four choices between a fixed amount A and a 50/50 gamble B

\begin{tabular}{lcc}
\hline Choice & Option $A$ & Option $B$ \\
\hline$(1)$ & $€ 24$ with $\mathrm{p}=1$ & $€ 45$ with $\mathrm{p}=0.5 ; € 0$ with $\mathrm{p}=0.5$ \\
$(2)$ if $(1)=\mathrm{A}$ & $€ 12$ with $\mathrm{p}=1$ & $€ 45$ with $\mathrm{p}=0.5 ; € 0$ with $\mathrm{p}=0.5$ \\
$(2)$ if $(1)=\mathrm{B}$ & $€ 36$ with $\mathrm{p}=1$ & $€ 45$ with $\mathrm{p}=0.5 ; € 0$ with $\mathrm{p}=0.5$ \\
$\ldots$ & $\ldots$ & $\ldots$ \\
See Figure A1 in Online Appendix A for all conditional sequences of choices. \\
\hline
\end{tabular}

EG Choice of preferred lottery from a menu of six 50/50 gambles

Option $1 € 21$ with $\mathrm{p}=0.5 ; € 21$ with $\mathrm{p}=0.5$

Option $2 € 27$ with $\mathrm{p}=0.5 ; € 18$ with $\mathrm{p}=0.5$

Option $3 € 33$ with $\mathrm{p}=0.5 ; € 15$ with $\mathrm{p}=0.5$

Option $4 € 39$ with $\mathrm{p}=0.5 ; € 12$ with $\mathrm{p}=0.5$

Option $5 € 45$ with $\mathrm{p}=0.5 ; € 9$ with $\mathrm{p}=0.5$

Option $6 \quad € 52$ with $\mathrm{p}=0.5 ; € 2$ with $\mathrm{p}=0.5$

HL Ten separate choices between two lotteries A and B

\begin{tabular}{ccc}
\hline Choice & Option $A$ & Option $B$ \\
$(1)$ & $€ 24$ with $\mathrm{p}=0.1 ; € 19.20$ with $\mathrm{p}=0.9$ & $€ 46.40$ with $\mathrm{p}=0.1 ; € 1.20$ with $\mathrm{p}=0.9$ \\
$(2)$ & $€ 24$ with $\mathrm{p}=0.2 ; € 19.20$ with $\mathrm{p}=0.8$ & $€ 46.40$ with $\mathrm{p}=0.2 ; € 1.20$ with $\mathrm{p}=0.8$ \\
$(3)$ & $€ 24$ with $\mathrm{p}=0.3 ; € 19.20$ with $\mathrm{p}=0.7$ & $€ 46.40$ with $\mathrm{p}=0.3 ; € 1.20$ with $\mathrm{p}=0.7$ \\
$(4)$ & $€ 24$ with $\mathrm{p}=0.4 ; € 19.20$ with $\mathrm{p}=0.6$ & $€ 46.40$ with $\mathrm{p}=0.4 ; € 1.20$ with $\mathrm{p}=0.6$ \\
$(5)$ & $€ 24$ with $\mathrm{p}=0.5 ; € 19.20$ with $\mathrm{p}=0.5$ & $€ 46.40$ with $\mathrm{p}=0.5 ; € 1.20$ with $\mathrm{p}=0.5$ \\
$(6)$ & $€ 24$ with $\mathrm{p}=0.6 ; € 19.20$ with $\mathrm{p}=0.4$ & $€ 46.40$ with $\mathrm{p}=0.6 ; € 1.20$ with $\mathrm{p}=0.4$ \\
$(7)$ & $€ 24$ with $\mathrm{p}=0.7 ; € 19.20$ with $\mathrm{p}=0.3$ & $€ 46.40$ with $\mathrm{p}=0.7 ; € 1.20$ with $\mathrm{p}=0.3$ \\
$(8)$ & $€ 24$ with $\mathrm{p}=0.8 ; € 19.20$ with $\mathrm{p}=0.2$ & $€ 46.40$ with $\mathrm{p}=0.8 ; € 1.20$ with $\mathrm{p}=0.2$ \\
$(9)$ & $€ 24$ with $\mathrm{p}=0.9 ; € 19.20$ with $\mathrm{p}=0.1$ & $€ 46.40$ with $\mathrm{p}=0.9 ; € 1.20$ with $\mathrm{p}=0.1$ \\
$(10)$ & $€ 24$ with $\mathrm{p}=1$ & $€ 46.40$ with $\mathrm{p}=1$ \\
\hline
\end{tabular}

GP Decision what fraction of $€ 24$ to invest in a project that pays 2.5 times the amount invested or 0 with equal probability.

$€ 24-€$ invested $+2.5 \times €$ invested, with $\mathrm{p}=0.5$

$€ 24-€$ invested, with $\mathrm{p}=0.5$

Notes: The table presents the gambles involved in the four risk preference elicitation tasks. Euro values stated refer to the parametrization in the private and professional investor sample. For subjects in the student sample, all values are divided by 4 . FA takes a value between 1 and 16 , according to the certainty equivalent resulting from the last of the four choices in the staircase risk task. EG is the rank (1-6) of the gamble chosen from a menu of six 50/50 gambles, increasing in risk. HL is the number of decision rows left after switching to the higher-risk lottery, ranging from 0 to 10 . GP is the EUR amount invested in the risky project and takes values between 0 and 6 . Investment amounts can be adjusted in steps of $€ 2$. Higher values imply higher risk tolerance across all four tasks. 


\section{Treatments}

We randomly assign subjects to one of two main incentive conditions. In the FLAT condition, subjects receive a fixed fee as a reward for participating in the experiment. The fixed participation fee is $€ 12$ for subjects in the private and professional investor samples, and is $€ 3$ in the student sample. Payment of the fixed participation fee is independent of the choices made in the experiment. In addition to the participation fee, subjects in the INCENTIVES condition are paid the earnings resulting from their choice in one experimental task, which is randomly selected at the end of the experiment. In case the selected task involves a series of decisions, a second random draw determines the decision to be paid out. Subjects are then paid according to their choice and the random outcome of the respective lottery.

Subjects in both conditions are presented with the same experimental tasks and experimental instructions (except for minor differences necessary to explain the payment protocols). At the beginning of the experiment, we explicitly inform subjects in the FLAT group that the payoffs of their decisions are hypothetical and do not affect their final payoff.

Note that as an intended consequence of this design, payoffs are lower in the FLAT condition than in the Incentives condition (see Table A1). To control for the sensitivity of our results to the absolute amount paid to subjects, we include a third incentive condition, FLAT_HIGH, for the student sample only (i.e., students were randomly allocated to one of the three treatments). Under this regime, student subjects receive a fixed participation fee equal to the average payout of students in the INCENTIVES condition, amounting to $€ 9$.

\section{Experimental Protocol}

To determine the target number of subjects to be recruited, we performed a power analysis following Cohen (1988) for behavioral sciences. We aim to maximize statistical power for each sample given the particular constraints with respect to recruitment possibilities. Applying the predefined target parameters to our realized sample sizes, our tests have 90\%-a priori power to detect effect sizes as low as $0.23,0.42$, and 0.32 in mean differences between the INCENTIVES and FLAT condition for the sample of private investors, professional investors, and students, respectively. These numbers are in the range of small and small-to-medium effect sizes, as suggested by Cohen (1988). We provide details on our power analysis in Table A5 in Online Appendix C.

The experiment was administered online using Limesurvey. We recruited subjects for participation via e-mail. Through e-mail, we recruited student participants from the University of Innsbruck using Hroot (Bock et al., 2014). In addition, we invited private investors from a panel of 2,000 clients of a large German brick-and-mortar bank who regularly participate in 
short online surveys/experiments administered by Goethe University Frankfurt. Third, we recruited professional investors via two channels. Two-thirds of the professional investor sample were recruited from the proprietary subject pool of professional investors (www.before.world) at the University of Innsbruck, some of whom had participated in previous unrelated studies. The remaining professionals are fund managers from different European countries whom we identified via their fund affiliation using data from Morningstar.

To avoid potential selection bias into either of the incentive conditions, we use a standardized invitation letter for all subjects. Subjects learn about the payment protocol relevant for them only upon starting the experiment.

\section{Data and sample characteristics}

We collected data for all subsamples in April and May 2020. Overall, 1,727 subjects completed the experiment. Once the experiment started, we offered unlimited time to finish to avoid exerting time pressure on subjects who were potentially engaged with risk elicitation experiments for the first time. However, to screen out participants who plausibly did not take the experiment seriously and to avoid potential noise due to outliers, we drop subjects in the top $(99 \%)$ and bottom percentiles $(1 \%)$ of the response time (34 respondents). ${ }^{7}$ The final sample consists of 1,693 subjects, comprising 821 subjects from the private investor sample, 244 from the financial professional investor sample, and 628 from the student sample. The median response time in the final sample is 13.22 minutes with a standard deviation of 9.88 minutes.

Panel A of Table A1 describes the private investor sample. Respondents in this sample are retail clients at a large German bank with a national branch network and are part of a regular online survey panel. ${ }^{8}$ Of the respondents, 26 percent are female, the average age is 53 years, and subjects' reported average net household income is $€ 4,292$. Of the private investors, 79 percent invest in stocks or stock mutual funds.

The private investor sample is well balanced along these characteristics across the two treatment arms, as indicated by the close averages and corresponding high p-values from a two-sided t-test of equal means in column 4 of Table A1 (Panel A) in the Online Appendix. The only significant difference between subjects in the various incentive conditions arises in

\footnotetext{
${ }^{7}$ Our results are robust to varying this cutoff, as well as applying the cutoff to the three subsamples individually. The propensity toward excessive response times does not systematically vary with the treatment condition.

${ }^{8}$ This panel consists of about 4,000 clients who signed up to regularly participate in online surveys for the purpose of academic research. For the present study, we selected 2,000 clients from the panel, over-sampling clients with an investment account (e.g. bonds, stocks, mutual funds) as opposed to clients holding a current account only. As private investor demographics have been elicited in an earlier survey wave, they are not re-elicited in the present study, a constraint that causes demographics to be missing for a small number of private investors who did not participate in this earlier survey.
} 
overall response time, which is significantly higher under the INCENTIVES condition. However, as we show below, this difference does not result from longer decision times in the single experimental tasks in the INCENTIVES condition, but is explained by subjects' need to spend more time reading longer texts outlining the payoff protocol in the INCENTIVES condition. This condition applies equally to all three subsamples.

Panel B of Table A1 describes the professional investors sample. Respondents in this sample are predominantly male ( 89 percent) and the average age is 42 years. The majority of professional investors are fund managers (35 percent), portfolio managers (18 percent), analysts (10 percent), and risk managers ( 7 percent). Again, the sample is well balanced across both treatment arms, as indicated by the close averages and corresponding high pvalues from a two-sided t-test in column 4 of Table A1 (Panel B) in the Online Appendix.

Panel C of Table A1 in the Online Appendix characterizes the student sample. Most subjects in the student sample are female (56 percent), and the average age is 24 years. Almost one in four student subjects invests in stocks or stock mutual funds. Both treatments in our student sample are well balanced along these characteristics. Given that the student sample has three treatment conditions - INCENTIVES, FLAT and FLAT_HIGH -, the p-value displayed in column 5 of Table A1 (Panel C) refers to a test regressing each characteristic on the treatment group indicators, with the null of all coefficients being jointly zero.

\section{Results}

\section{Risk-taking}

Result 1: Risk-taking of private investors, professional investors, and students does not differ across incentive conditions in three of the four experimental tasks. In the HL task, professional investors and students engage in slightly less risk-taking in the INCENTIVES than in the FLAT condition.

Support: We start our analysis by comparing mean choices in the four experimental tasks between subjects in the FLAT and INCENTIVES condition. The upper panel in Figure 1 displays average choices by experimental task, incentive condition, and subject pool. For FA, we display a value between 1 and 16 - according to ordinal ranking of the resulting certainty equivalent - with higher values indicating higher levels of risk tolerance. For EG, we display the rank (1 to 6 ) of the gamble chosen from the menu of six 50/50 gambles, with higher values indicating higher risk tolerance. In the HL-task, we present the number of decision rows left after switching to the higher-risk lottery, ranging from 0 to 10 . Higher values indicate higher risk tolerance. For GP, we show the euro amount invested in the risky investment. To make choices comparable across subject pools, we divide the amount invested in the GP-task in 
the private and professional investor subsamples by four. GP hence takes on values between 1 and 6 , with higher values again indicating higher risk tolerance. ${ }^{9}$ The light (dark) shaded bars represent subjects' choices in the FLAT (INCENTIVES) condition. We report the p-values of two-sided t-tests for equality of mean choices in Table A2 in the Online Appendix.

Figure 1: Effect of task-related incentives on risk-taking
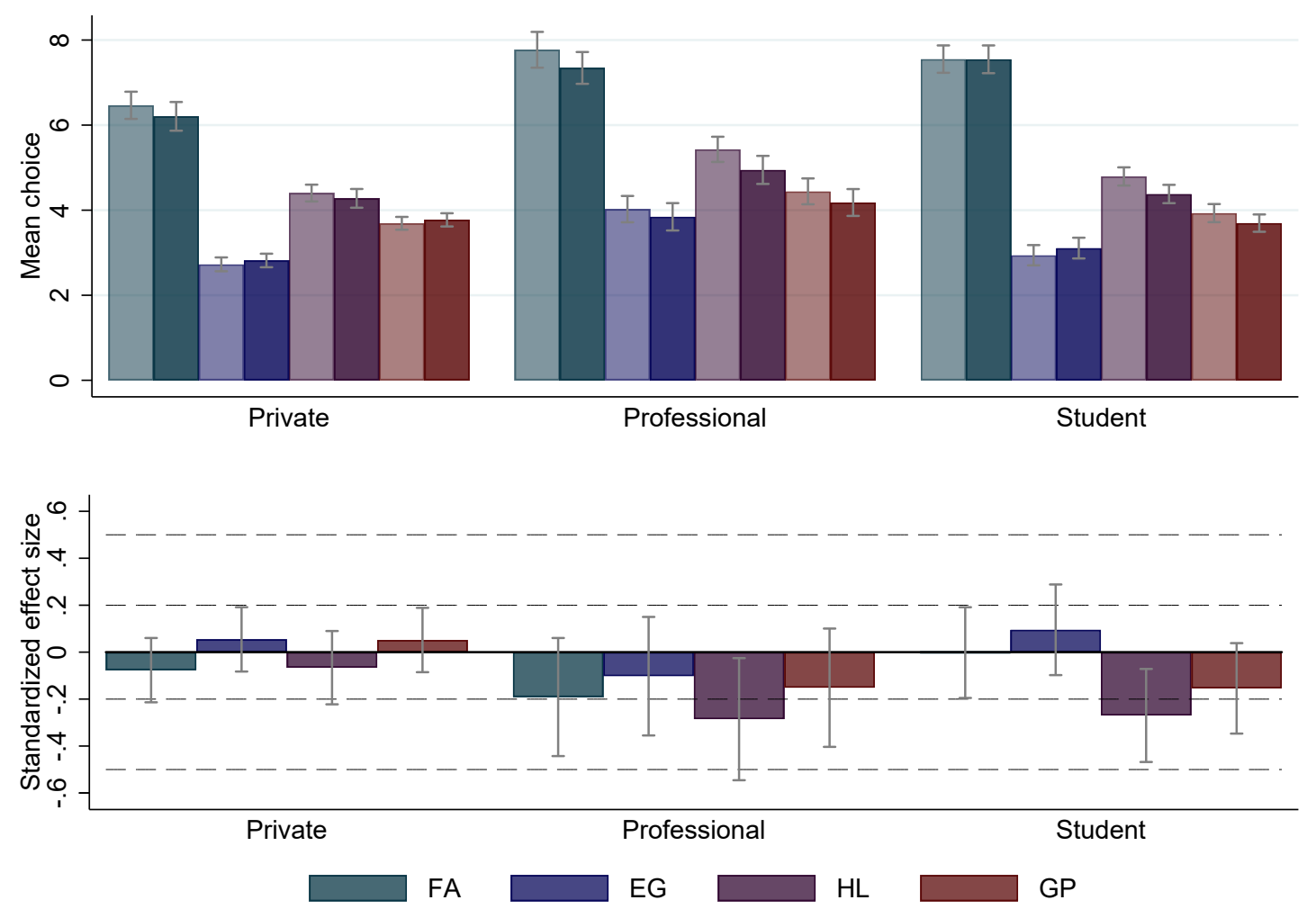

Notes: Upper panel: Mean choices by subject pool (3 blocks), task (4 colors), and incentive condition (2 shades). The light (dark) shaded bars represent the choices of subjects in the FLAT (INCENTIVEs) condition. For all tasks, higher levels indicate greater risk-taking. Owing to the different nature of the underlying tasks, the absolute height of the bars cannot be compared across tasks. FA takes a value between 1 and 16, according to the ordinal rank of the certainty equivalent resulting from the last of the four choices in the staircase risk task. EG is the rank (1-6) of the gamble chosen from a menu of six 50/50 gambles, increasing in risk. HL is the number of decision rows left after switching to the higher-risk lottery, ranging from 0 to 10 . GP is the euro amount invested in the risky project and takes values between 0 and 24 for private and professional investors, and values between 0 and 6 for students. For illustration purposes in the above graph, we align these values across samples by dividing the invested amount in the private and professional investor sample by 4 . Lower panel: Standardized treatment effects of incentivization on risk-taking, by subject pool and task. Bars represent the coefficient of an indicator variable for whether a subject has been assigned to the INCENTIVES condition in regressions with standardized choices. We standardize choices by deducting the mean and dividing by the standard deviation in the respective subject pool. Error bars indicate $95 \%$-confidence intervals.

As illustrated in the upper panel of Figure 1 and confirmed by the test statistics in

\footnotetext{
${ }^{9}$ Investment amounts can be adjusted in steps of $€ 0.50(€ 2.00)$.
} 
Table A2, we find no significant differences between subjects' choices in the various incentive conditions in three of the four experimental tasks. In the case of HL, we find that subjects' behavior is slightly more risk-averse in the INCENTIVES condition than under the FLAT regime in the student sample ( $\mathrm{t}$-test, $\mathrm{p}$-value $=0.008, \mathrm{~N}=388$ ) and the professional investor sample (t-test, $\mathrm{p}$-value $=0.032, \mathrm{~N}=226$ ).

In the lower panel of Figure 1, we display standardized treatment effects sizes, along with $95 \%$ confidence intervals. Within each subject pool, we standardize the choices in the different tasks by subtracting the mean and dividing by the standard deviation of the distribution of choices in the respective subject pool. We then regress standardized choices on an indicator variable for whether a subject has been assigned to the INCENTIVES condition. The figure confirms the above finding and illustrates the relative magnitude of the decrease in risk-taking in the HL task on the part of finance professionals and students in the INCENTIVES condition. In both cases, the difference amounts to .27 of a standard deviation.

Figure A2 in the Online Appendix reproduces Figure 1 comparing choices in the student sample by incentive condition, including the third condition FLAT_HIGH. We find that the absolute level of the fixed participation fee does not alter our results. Student subjects behave virtually identically under the FLAT_HIGH and FLAT condition, as illustrated by the rightmost set of bars. Again, student subjects in the INCENTIVES condition take risks similar to those in both flat fee conditions FLAT_HIGH or FLAT in the FA, EG, and GP tasks. The result that subjects in the student sample take less risk under the INCENTIVES regime than when receiving a fixed fee only holds irrespective of the absolute height of the fixed amount, as illustrated by the set of bars in the middle and on the left.

In addition to comparing the means, we also compare the distributions of individuals' choices in each task by incentive condition, separately for each subject pool (Figure A3 in the Online Appendix). ${ }^{10}$ In support of Result 1, we find that Kolmogorov-Smirnov tests fail to reject the null hypothesis of equal distributions under both incentive regimes across tasks and subject pools, with the only exception being the distributions of the switching point in the HL task in the professional investor sample (KS-test, $\mathrm{p}$-value $=0.022, \mathrm{~N}=226$; p-values of KS-tests are reported in Table A2 in the Online Appendix). ${ }^{11}$

\footnotetext{
${ }^{10}$ Panel A displays choices of private investors, and Panels $\mathrm{B}$ and $\mathrm{C}$ refer to professional investors and students, respectively. Light (dark) colored bars represent the choices of subjects in the FLAT (INCENTIVES) condition.

${ }^{11}$ As an additional test on the role of incentives for decision-making, we compare the probability of extreme choices for each task by incentive condition. To do so, we define an indicator equal to one if a respondent selects into the lowest or highest risk tolerance category, according to the respective measure. In Figure A4 in the Online Appendix, we illustrate that the propensity to provide an extreme response does not significantly differ by incentive condition at conventional significance levels of 5 percent or higher across all three subject pools, according to two-sided t-tests (see Table A3 in the Online Appendix).
} 


\section{Incentives and effort}

Result 2: In general, effort - as measured by decision times of subjects - does not differ across incentive conditions, tasks, and subject pools. Moreover, while drop-out rates in the experiment differ considerably across subject pools, results reveal no systematic in the propensity to drop out by incentive condition.

Figure 2: Effect of task-related incentives on decision times

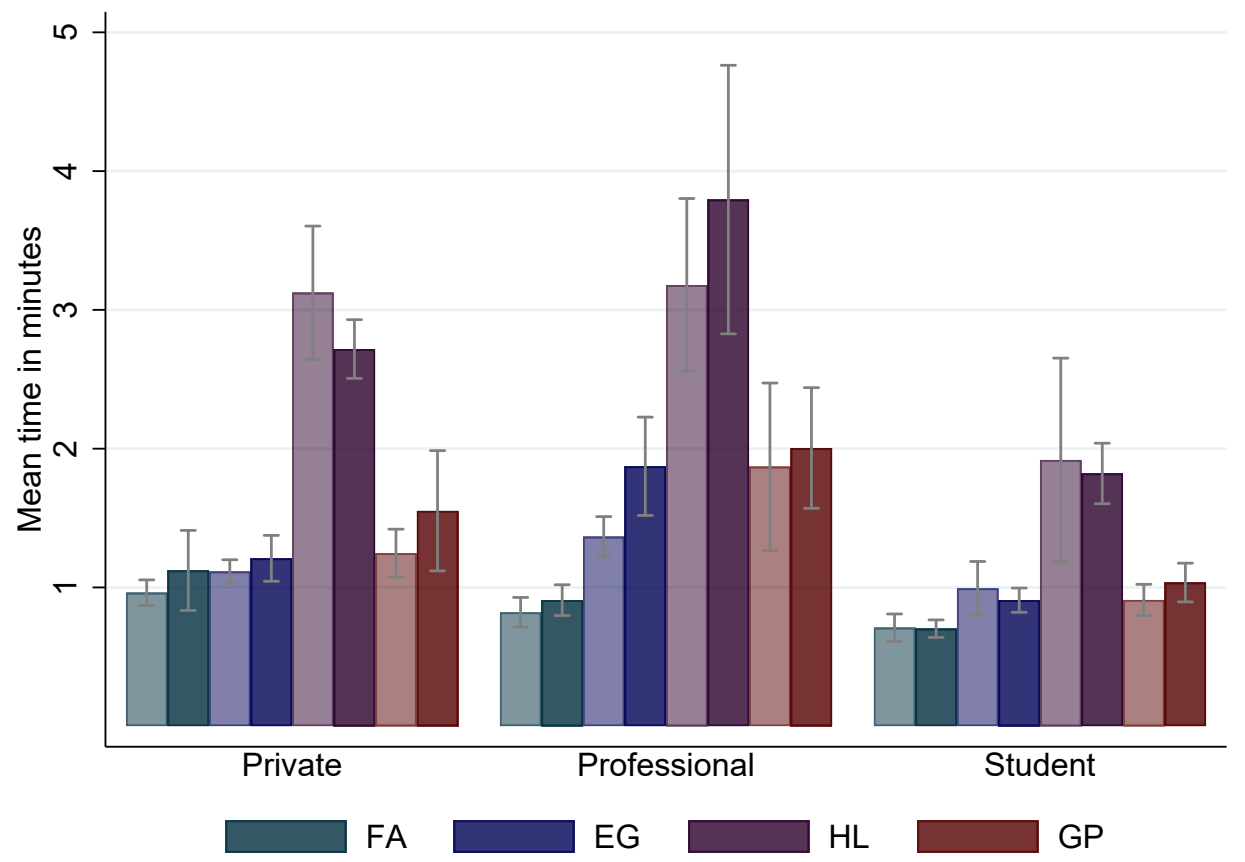

Notes: The figure compares the average decision times in minutes for the four experimental tasks (4 colors) by incentive condition ( 2 shades) separately for the three subject pools ( 3 blocks). The light (dark) shaded bars represent the choices of subjects in the FLAT (INCENTIVES) condition. Error bars indicate $95 \%$ confidence intervals.

Support: Another dimension along which task-related incentives may affect subjects' behavior in the experiments is the effort subjects apply in making decisions. We follow the literature in using decision time when making their choices as a measure of effort (Wilcox, 1993; Camerer and Hogarth, 1999) and we compare decision times in the four tasks by incentive condition. Subjects take on average 0.88 minutes to complete the FA, 1.13 minutes for the EG, 2.58 minutes for the HL, and 1.29 minutes for the GP task. As illustrated in Figure 2 , we find no systematic differences in decision times by incentive condition across tasks and subject pools, except for professional investors in the INCENTIVES condition of the EG task (t-test, $\mathrm{p}$-value $=0.01, \mathrm{~N}=244$; see Table A4 in the Online Appendix). ${ }^{12}$ These results imply

\footnotetext{
${ }^{12}$ The difference of 0.50 minutes is somewhat surprising given the relative simplicity of the task and suggests it may be due to outliers.
} 
that the significantly higher total time spent in the experiment observed for subjects in the INCENTIVES condition across the three subject pools (see Table A1 in the Online Appendix) results from subjects taking more time to read the details of the task-related payoff protocol rather than from spending more time thinking about their choices.

Figure 3: Drop-out rates and inconsistent choices

Panel A: Drop-out rates
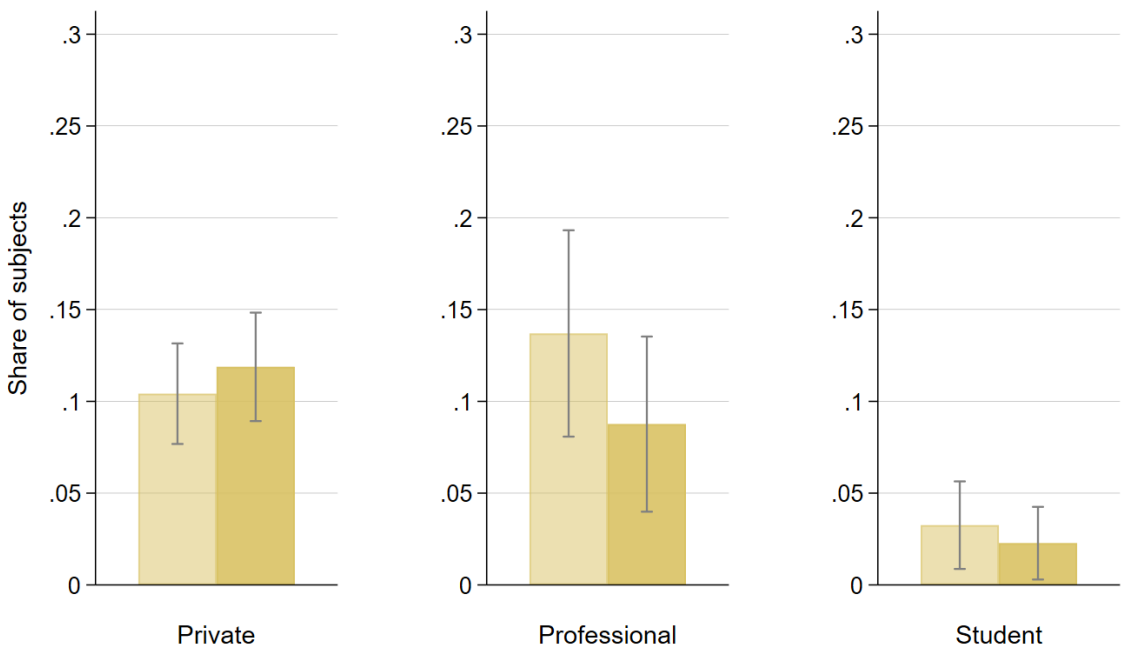

Panel B: Multiple switchers (HL)
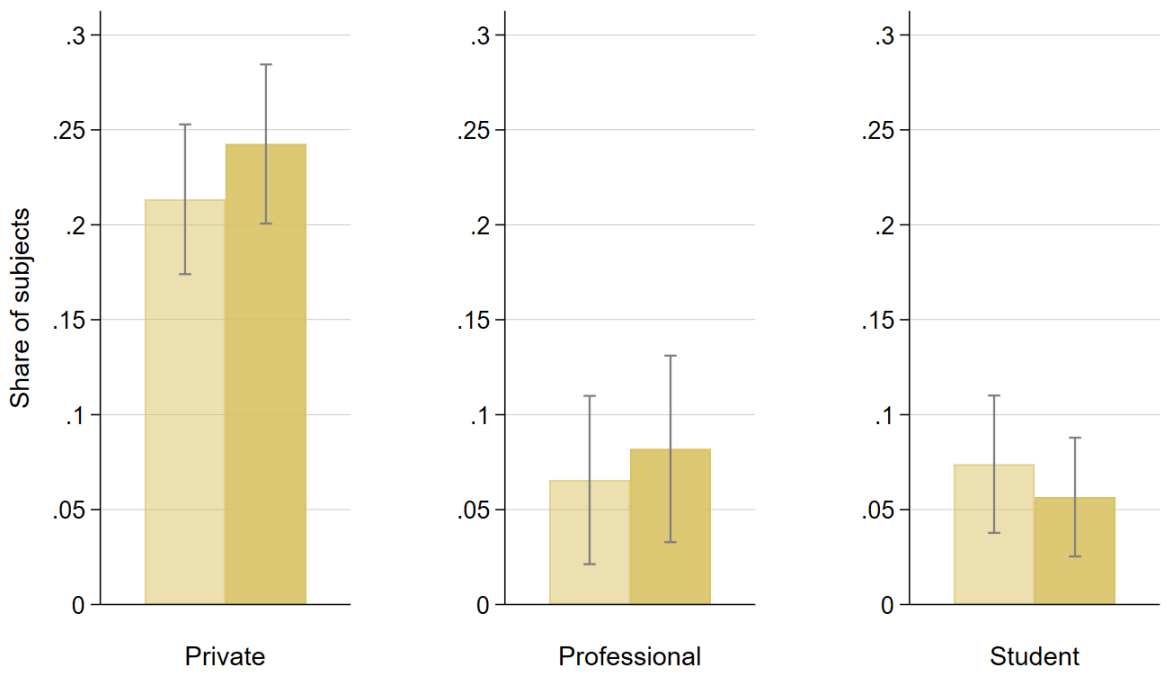

Flat Incentives

Notes: The figure shows the propensity of respondents to drop out from the experiment (Panel A) and the share of subjects with inconsistent answers (multiple switching points) in the HL task (Panel B) by incentive condition, separately for the three subject pools. Drop out rates are calculated based on the overall number of respondents who started the experiment $(\mathrm{N}=1,882)$, of which 1,727 completed it. Error bars indicate 95\%-confidence intervals. 
As a second proxy for individuals' effort, we test whether task-related incentives act to increase subjects' perseverance, reducing the number of participants who exit the experiment prior to completion. Overall, 1,882 subjects started the experiment, of which 1,727 completed it, translating into a total drop-out rate of 8 percent. We find that drop-out rates differ considerably across subject pools and are substantially lower in the student sample (below 3 percent) than in the private investor and professional investor samples (about 11 percent in both samples). However, in all subject pools, we do not find systematic differences in the propensity to drop out from the experiment across incentive conditions (see Panel A of Figure 3 and Table A4 in the Online Appendix).

Finally, we test whether task-related incentives reduce instances of inconsistent behavior in the arguably complex HL task. In the HL task, we allow participants to switch between options $\mathrm{A}$ and $\mathrm{B}$ as they move down the 10 decision rows. This opportunity may result in inconsistent behavior as subjects could switch between options more than once. ${ }^{13}$ Across all subject pools, 15 percent of participants make inconsistent choices, which compares rather favorably to other studies (Crosetto and Filippin, 2016; Charness et al., 2013). The prevalence of inconsistent choices differs greatly across subject pools (see Panel B of Figure 3 and Table A4 in the Online Appendix). While the choices of about 7 percent of subjects in the student and professional investor sample produce multiple switching points, 23 percent of respondents in the private investor sample show this kind of behavior. ${ }^{14}$ Importantly, we find no evidence that the incentive regime does not affect the propensity to behave inconsistently in the HL task across the three subject pools.

\section{Incentives and consistency in risk-taking across tasks}

Result 3: As measured by the within-subject standard deviation of an individual's standardized choices, within-subject consistency of risk-taking across the four experimental tasks is unaffected by the incentive condition.

Support: As a last test, we investigate whether the incentive regime affects the withinsubject consistency of risk-taking across the four experimental tasks. To calculate the withinsubject standard deviation, we first standardize each subject's choices in the four experimental tasks by subtracting the mean and dividing by the standard deviation of the distribution of choices in the respective task and in the relevant subject pool. For each subject, we

\footnotetext{
${ }^{13}$ Remember, a risk-averse agent should start out by choosing option $\mathrm{A}$ in the first decision, and then switch to option $\mathrm{B}$ at some point before the last decision.

${ }^{14}$ One obvious reason lies in different education levels among the three subject pools. We find the propensity to give inconsistent answers in the private investor sample to be negatively correlated with educational achievement and financial literacy. Among private investors who have completed a college degree $(\mathrm{N}=401)$, the share of respondents giving inconsistent answers amounts to 13 percent. Accordingly, Dave et al. (2010) show that less sophisticated subjects have trouble understanding the HL protocol.
} 
Figure 4: Within-subject consistency in risk-taking across tasks

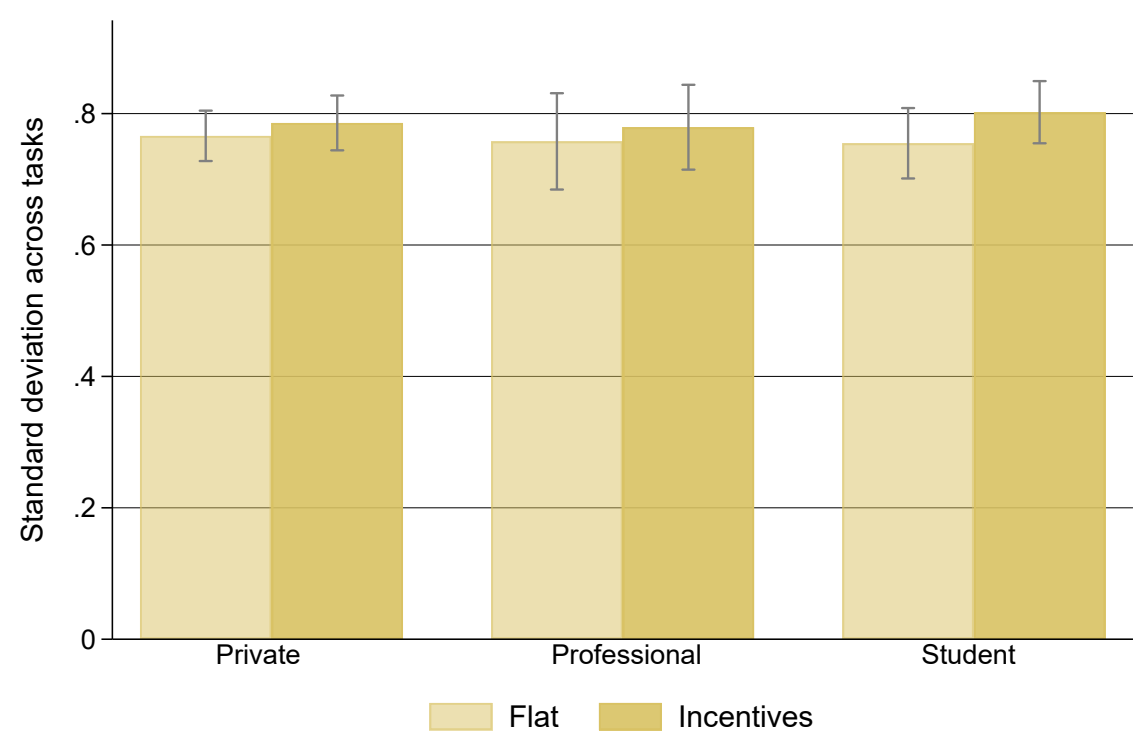

Notes: The figure compares the mean within-subject standard deviation for the four experimental tasks by incentive condition separately for the three subject pools. We standardize choices in the single tasks by deducting the mean and dividing by the standard deviation of choices made in the given task in the relevant subject pool. We then calculate the within-subject standard deviation over a subject's four standardized choices. The light (dark) shaded bars refer to subjects in the FLAT (InCEnTIVes) condition. Error bars indicate $95 \%$-confidence intervals.

then calculate the standard deviation over the subject's standardized choices in the four tasks. We do not find any evidence that task-related incentives affect the within-subject standard deviation of choices across the three subject pools (see Figure 4 and Table A4 in the Online Appendix). Interestingly, the within-subject standard deviation decreases strongly when excluding the HL task from the consistency measure (see Figure A5 in the Online Appendix). Singular exclusion of any of the other three choices does not produce a similar effect.

\section{Conclusion}

We use a systematic, large-sample approach with three subject pools of private investors, professional investors, and students, to investigate the impact of task-related monetary incentives on risk preferences, elicited in four standard experimental tasks: the staircase procedure by Falk et al. (2016, 2018), the gamble-choice task by Eckel and Grossman (2002), the investment game by Gneezy and Potters (1997), and the paired lottery choice task by Holt and Laury (2002). We find no significant differences between the choices of subjects in the different incentive conditions in 10 of the 12 in-sample comparisons across subject pools. Only in the 
Holt and Laury (2002) task do professional investors and students behave in a slightly more risk averse manner under a task-related incentive regime than under a regime where subjects receive a flat fee for participation. These results do not change when absolute differences in payment amounts are accounted for. We also find no systematic differences across incentive conditions with respect to task-specific response times, drop-out rates, inconsistent choice behavior, and within-subject consistency in risk-taking across tasks.

Our analyses so far remain silent as to why the HL task produces the only significant differences between monetarily incentivized and hypothetical choices. Our results show that inclusion of the HL choice increases within-subject consistency in choices across the four experimental tasks, providing evidence that individuals tend to behave "differently" in this task. Future research could tackle questions as to whether incentives matter more in complex tasks or whether incentives interact with specific features of the tasks, such as the ability to capture risk-seeking behavior, which is inherent to HL, but absent in other tasks (e.g., EG). ${ }^{15}$

Importantly, our results do not necessarily extend to experimental tasks other than the risk-preference elicitation tasks covered. For example, real task-related incentives have been shown to matter in valuation tasks, where subjects regularly overstate their valuation of alternatives or objects if choices are only hypothetical (List and Gallet, 2001). Similarly, numerous studies show that purely hypothetical tasks overstate socially desirable behaviors in subjects, such as altruism, cooperativeness, and patience (see Camerer and Mobbs (2017) for a recent review). In these settings, incentives are an integral part of the experimental design and it is hard to doubt the necessity to incentivize these tasks (Bardsley et al., 2020). We also do not argue that experimental subjects need not be paid at all. While some people may be intrinsically motivated to participate and will respond truthfully to experimental tasks (Read, 2005), payment of a flat reward for participation plausibly increases the willingness to participate and may help reduce selection into participation. As we paid all subjects a fixed reward for participation, we can only hypothesize about the potential effects of fixed participation fees.

Given the importance of risk preferences to researchers, policymakers, and industry professionals, future research must strive to settle the current methodological issues in the elicitation of individuals' attitudes toward risk. This obligation holds for large-scale labin-the-field experiments, where preferences are often elicited only as a control variable and where time and money are scarce. It also holds for applied settings, most prominently the elicitation of risk preferences as part of the financial advisory process under MiFID II (see

\footnotetext{
${ }^{15}$ See Crosetto and Filippin (2016) for a recent discussion of how characteristics of risk elicitation tasks such as complexity, availability of a safe option, or the range of risk attitudes may affect measured behaviors. To date, the literature contains no discussion of how these factors may interact with the presence or absence of task-related incentives.
} 
https://www.esma.europa.eu/policy-rules/mifid-ii-and-mifir; retrieved July 1, 2020). ${ }^{16}$ Thus, having lean experimental protocols that produce accurate measures of individuals' risk preferences is critical. In weighing complexity of the experimental design against the accuracy of preference measures, an important criterion is that of learning more about potential hypothetical bias in standard risk elicitation tasks. Our results imply that the degree of hypothetical bias is very limited in experiments administered online to private and professional investors (with non-task-related incentives).

\footnotetext{
${ }^{16}$ More generally, several studies have tested the power of preferences using standard experimental tasks to explain individuals' risky behaviors in the lab and in the field. In a recent study, Charness et al. (2020) show that incentivized standard measures of risk preferences are found to have very limited external validity (i.e., field behavior is measured with financial, health, and employment decisions in a representative Dutch population). In addition, those authors show that simpler measures perform better than the more complex measures.
} 


\section{References}

Alevy, Jonathan E, Michael S Haigh, John A List. 2007. Information cascades: Evidence from a field experiment with financial market professionals. Journal of Finance 62(1) 151-180.

Barberis, Nicholas, Ming Huang, Tano Santos. 2001. Prospect theory and asset prices. The Quarterly Journal of Economics 116(1) 1-53.

Bardsley, Nicholas, Robin Cubitt, Graham Loomes, Peter Moffatt, Chris Starmer, Robert Sugden. 2020. Experimental Economics: Rethinking the Rules. Princeton University Press, Princeton and London.

Beattie, Jane, Graham Loomes. 1997. The impact of incentives upon risky choice experiments. Journal of Risk and Uncertainty 14(2) 155-168.

Bock, Olaf, Ingmar Baetge, Andreas Nicklisch. 2014. hroot: Hamburg registration and organization online tool. European Economic Review 71 117-120. doi: 10.1016/j.euroecorev.2014.07.003.

Camerer, Colin. 1995. Individual decision making. Handbook of Experimental Economics .

Camerer, Colin, Dean Mobbs. 2017. Differences in behavior and brain activity during hypothetical and real choices. Trends in Cognitive Sciences 21(1) 46-56.

Camerer, Colin F, Robin M Hogarth. 1999. The effects of financial incentives in experiments: A review and capital-labor-production framework. Journal of Risk and Uncertainty 19(1-3) $7-42$.

Charness, Gary, Thomas Garcia, Theo Offerman, Marie Claire Villeval. 2020. Do measures of risk attitude in the laboratory predict behavior under risk in and outside of the laboratory? Journal of Risk and Uncertainty forthcoming.

Charness, Gary, Uri Gneezy, Alex Imas. 2013. Experimental methods: Eliciting risk preferences. Journal of Economic Behavior \& Organization 8743 - 51.

Cohen, Jacob. 1988. Statistical Power Analysis for the Behavioral Sciences. 2nd ed. Lawrence Erlbaum Associates, Hillsdale, NJ.

Crosetto, Paolo, Antonio Filippin. 2016. A theoretical and experimental appraisal of four risk elicitation methods. Experimental Economics 19(3) 613-641.

Dave, Chetan, Catherine C Eckel, Cathleen A Johnson, Christian Rojas. 2010. Eliciting risk rreferences: When is simple better? Journal of Risk and Uncertainty 41(3) 219-243. 
Dohmen, Thomas, Armin Falk, David Huffman, Uwe Sunde, Jürgen Schupp, Gert G. Wagner. 2011. Individual risk attitudes: Measurement, determinants, and behavioral consequences. Journal of the European Economic Association 9(3) 522-550.

Eckel, Catherine C., Philip J. Grossman. 2002. Sex differences and statistical stereotyping in attitudes toward financial risk. Evolution and Human Behavior 23(4) 281 - 295. doi: 10.1016/S1090-5138(02)00097-1.

Etchart-Vincent, Nathalie, Olivier l'Haridon. 2011. Monetary incentives in the loss domain and behavior toward risk: An experimental comparison of three reward schemes including real losses. Journal of Risk and Uncertainty 42(1) 61-83.

Falk, Armin, Anke Becker, Thomas Dohmen, David Huffman David, Uwe Sunde. 2016. The preference survey module: A validated instrument for measuring risk, time, and social preferences Working Paper.

Falk, Armin, Anke Becker, Thomas Dohmen, Benjamin Enke, David Huffman, Uwe Sunde. 2018. Global Evidence on Economic Preferences. The Quarterly Journal of Economics 133(4) 1645-1692.

Filippin, Antonio, Paolo Crosetto. 2016. A reconsideration of gender differences in risk attitudes. Management Science 62(11) 3138-3160. doi:10.1287/mnsc.2015.2294.

Gneezy, Uri, Alex Imas, John List. 2015. Estimating individual ambiguity aversion: A simple approach Working Paper.

Gneezy, Uri, Jan Potters. 1997. An experiment on risk taking and evaluation periods. The Quarterly Journal of Economics 112(2) 631-645. doi:10.1162/003355397555217.

Guiso, Luigi, Paolo Sodini. 2013. Household finance: An emerging field. George Constantinides, Milton Harris, René Stulz, eds., Handbook of the Economics of Finance, vol. 2. Elsevier, 1397-1532.

Haigh, Michael S, John A List. 2005. Do Professional Traders Exhibit Myopic Loss Aversion? An Experimental Analysis. Journal of Finance 60(1) 523-534.

Harrison, Glenn W. 1994. Expected utility theory and the experimentalists. John D Hey, ed., Experimental Economics. Springer, 43-73.

Harrison, Glenn W. 2006. Hypothetical bias over uncertain outcomes. John A List, ed., Using Experimental Methods in Environmental and Resource Economics. The name of the publisher, Northampton, MA, 201-213. 
Harrison, Glenn W, Eric Johnson, Melayne M McInnes, E Elisabet Rutström. 2005. Risk aversion and incentive effects: Comment. American Economic Review 95(3) 897-901.

Hertwig, Ralph, Andreas Ortmann. 2001. Experimental practices in economics: A methodological challenge for psychologists? Behavioral and Brain Sciences 24(3) 383-403.

Holt, Charles A., Susan K. Laury. 2002. Risk aversion and incentive effects. American Economic Review 92(5) 1644-1655. doi:10.1257/000282802762024700.

Holt, Charles A, Susan K Laury. 2005. Risk aversion and incentive effects: New data without order effects. American Economic Review 95(3) 902-912.

Kahneman, Daniel, Amos Tversky. 1979. Prospect theory: An analysis of decision under risk. Econometrica 47(2) 263-291.

Kirchler, Michael, Florian Lindner, Utz Weitzel. 2018. Rankings and risk-taking in the finance industry. Journal of Finance 73(5) 2271-2302.

Kuehberger, Anton, Michael Schulte-Mecklenbeck, Josef Perner. 2002. Framing decisions: Hypothetical and real. Organizational Behavior and Human Decision Processes 89(2) 1162 $-1175$.

List, John, Craig Gallet. 2001. What experimental protocol influence disparities between actual and hypothetical stated values? Environmental $\&$ Resource Economics 20(3) 241254 .

List, John A. 2003. Does market experience eliminate market anomalies? Quarterly Journal of Economics 118(1) 41-71.

Markowitz, Harry. 1952. Portfolio selection. The Journal of Finance 7(1) 77-91.

Merton, Robert C. 1969. Lifetime portfolio selection under uncertainty: The continuous-time case. Review of Economics and Statistics 247-257.

Pratt, John. 1964. Risk aversion in the small and in the large. Econometrica 32(1-2) 122-136.

Read, Daniel. 2005. Monetary incentives, what are they good for? Journal of Economic Methodology 12(2) 265-276.

Smith, Vernon L. 1976. Experimental economics: Induced value theory. American Economic Review 66(2) 274-279.

Smith, Vernon L., James M. Walker. 1993. Monetary rewards and decision costs in experimental economics. Economic Inquiry 31(2) 245-261. 
Wilcox, Nathaniel T. 1993. Lottery choice: Incentives, complexity and decision time. Economic Journal 103(421) 1397-1417. 
Online Appendix: On the (ir)relevance of monetary incentives in risk preference elicitation

Andreas Hackethal ${ }^{12}$, Michael Kirchler ${ }^{3}$, Christine Laudenbach ${ }^{1}$, Michael Razen ${ }^{3}$, Annika Weber ${ }^{12}$

\section{A Additional Figures}

Figure A1: Sequence of choices in the staircase procedure FA

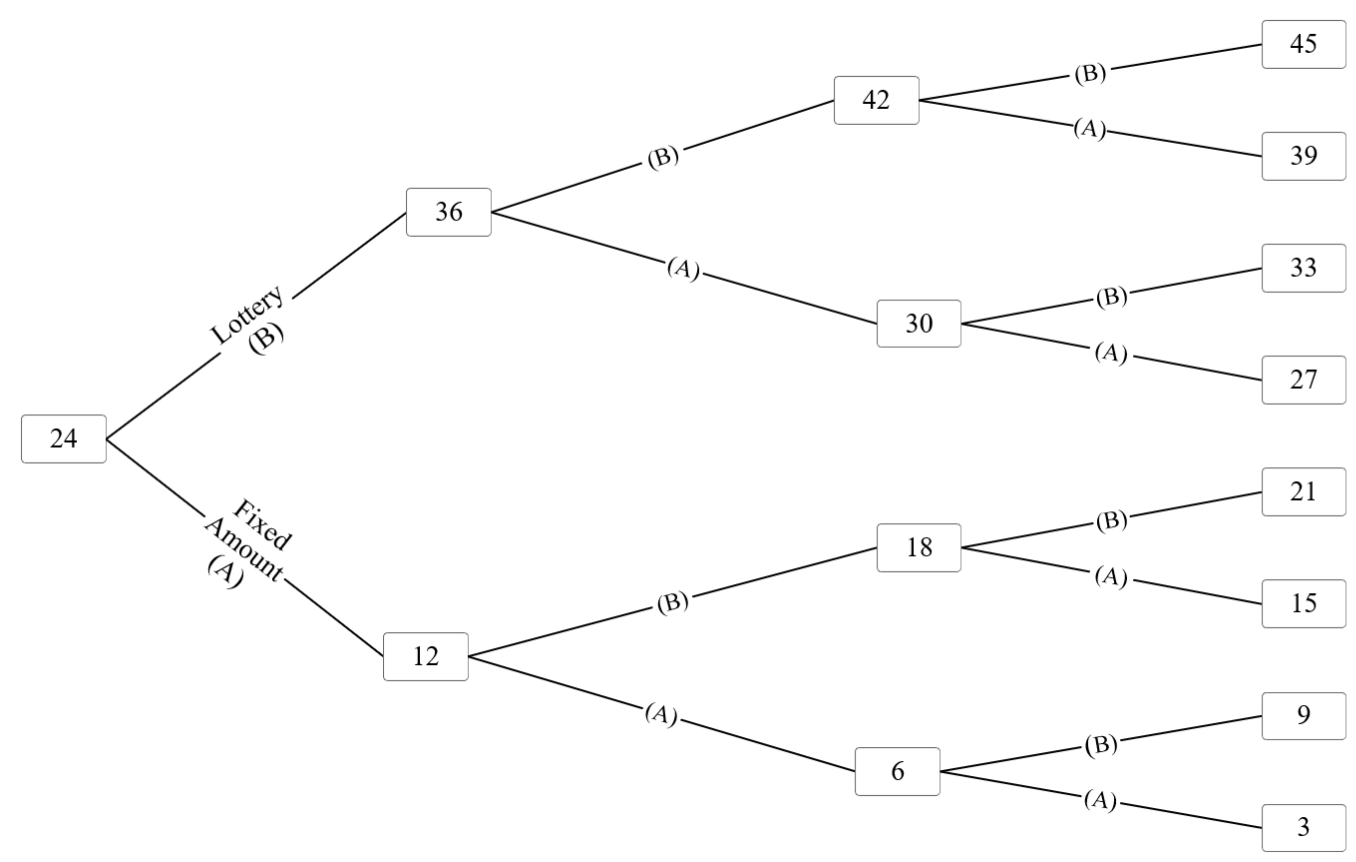

Notes: The staircase procedure following FA works as follows. First, each respondent was asked whether they would prefer to receive 24 EUR for sure or whether they preferred a 50:50 chance of receiving 45 EUR or nothing. In case the respondent opted for the fixed amount (A), the fixed amount being offered in the second question decreased to 12 EUR. If, on the other hand, the respondent opted for the lottery (B), the safe amount was increased to 36 . Working further through the tree follows the same logic.

\footnotetext{
${ }^{1}$ Goethe University Frankfurt

${ }^{2}$ Leibniz Institute for Financial Research SAFE, Frankfurt

${ }^{3}$ University of Innsbruck
} 
Figure A2: Effect of task-related incentives on risk-taking - Student sample
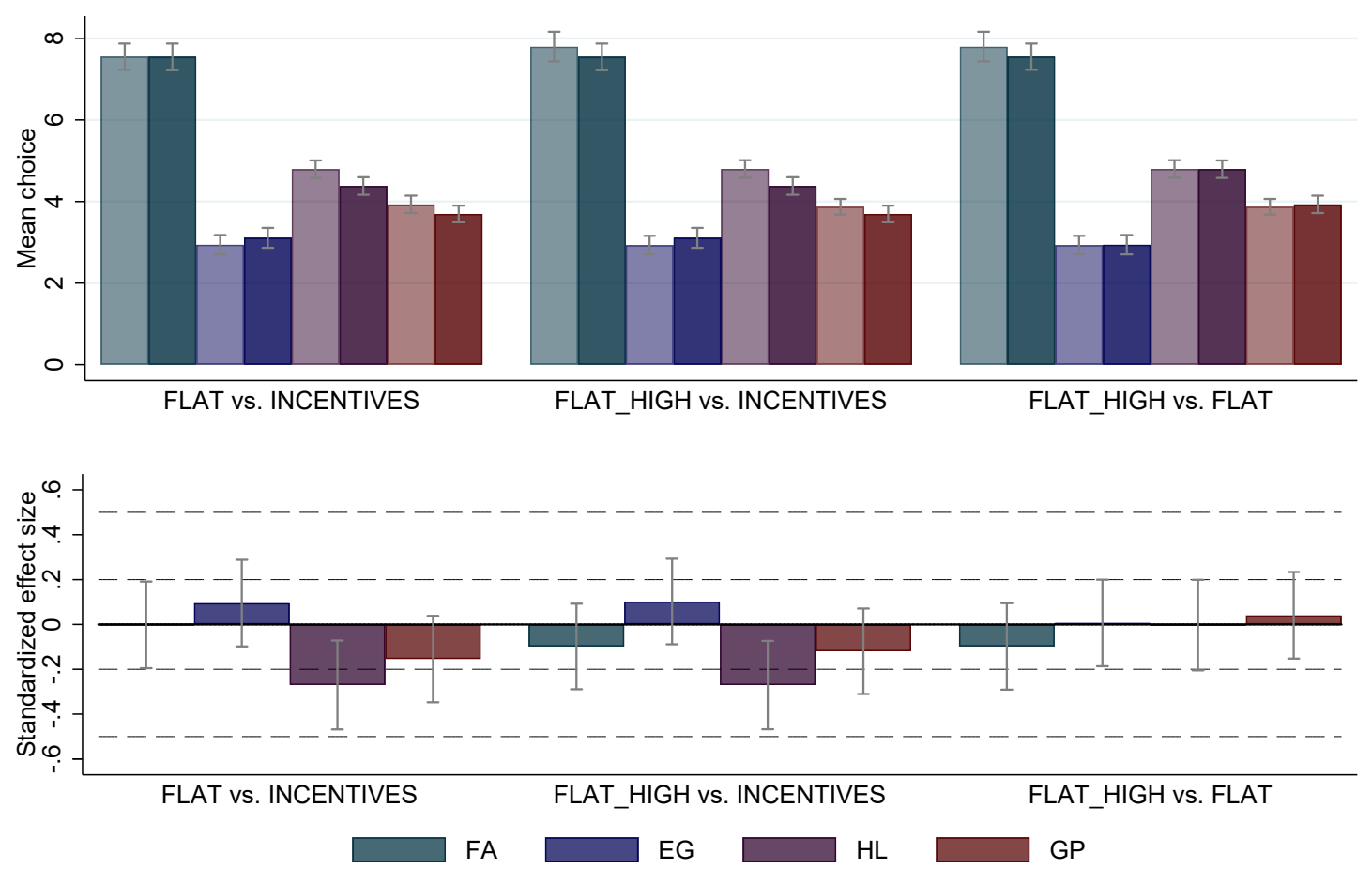

Notes: The upper panel shows pairwise comparisons of the mean choices in the four different tasks (FA in emerald, EG in blue, HL in purple, and GP in maroon) across subjects in the three different incentive conditions in the student subject pool. The left block compares subjects in the FLAT condition (light shaded bars) to subjects in the INCENTIVES condition (dark shaded bars). The middle block compares subjects in the FLAT_HIGH condition (light shaded bars) to subjects in the INCENTIVES condition (dark shaded bars). The right block compares subjects in the FLAT_HIGH condition (light shaded bars) to subjects in the FLAT condition (dark shaded bars). Subjects in the FLAT (FLAT_HIGH) condition receive a fixed participation fee of $€ 3$ (€9). Subjects in the INCENTIVES condition receive a fixed participation fee of $€ 3$ plus the earnings resulting from their choice in one randomly determined task. FA takes a value between 1 and 16, according to the certainty equivalent resulting from the last of the four choices in the staircase risk task. EG is the rank (1-6) of the gamble chosen from a menu of six 50/50 gambles, increasing in risk. HL is the number of decision rows left after switching to the higher-risk lottery, ranging from 0 to 10. GP is the EUR amount invested in the risky project and takes values between 0 and 6 . Investment amounts can be adjusted in steps of $€ 0.50$. Higher values imply higher risk tolerance across all four tasks. The lower panel shows standardized treatment effects. We standardize the choices of student subjects in the four different task by subtracting the mean and dividing by the standard deviation of the distribution of choices in the subject pool. We then regress standardized choices on an indicator for whether a subject has been assigned to the comparison incentive condition (INCENTIVES for the left and middle blocks, FLAT for the right block). Error bars indicate 95\%-confidence intervals. 
Figure A3: Effect of task-related incentives on risk-taking - Distributions

Panel A: Private investors
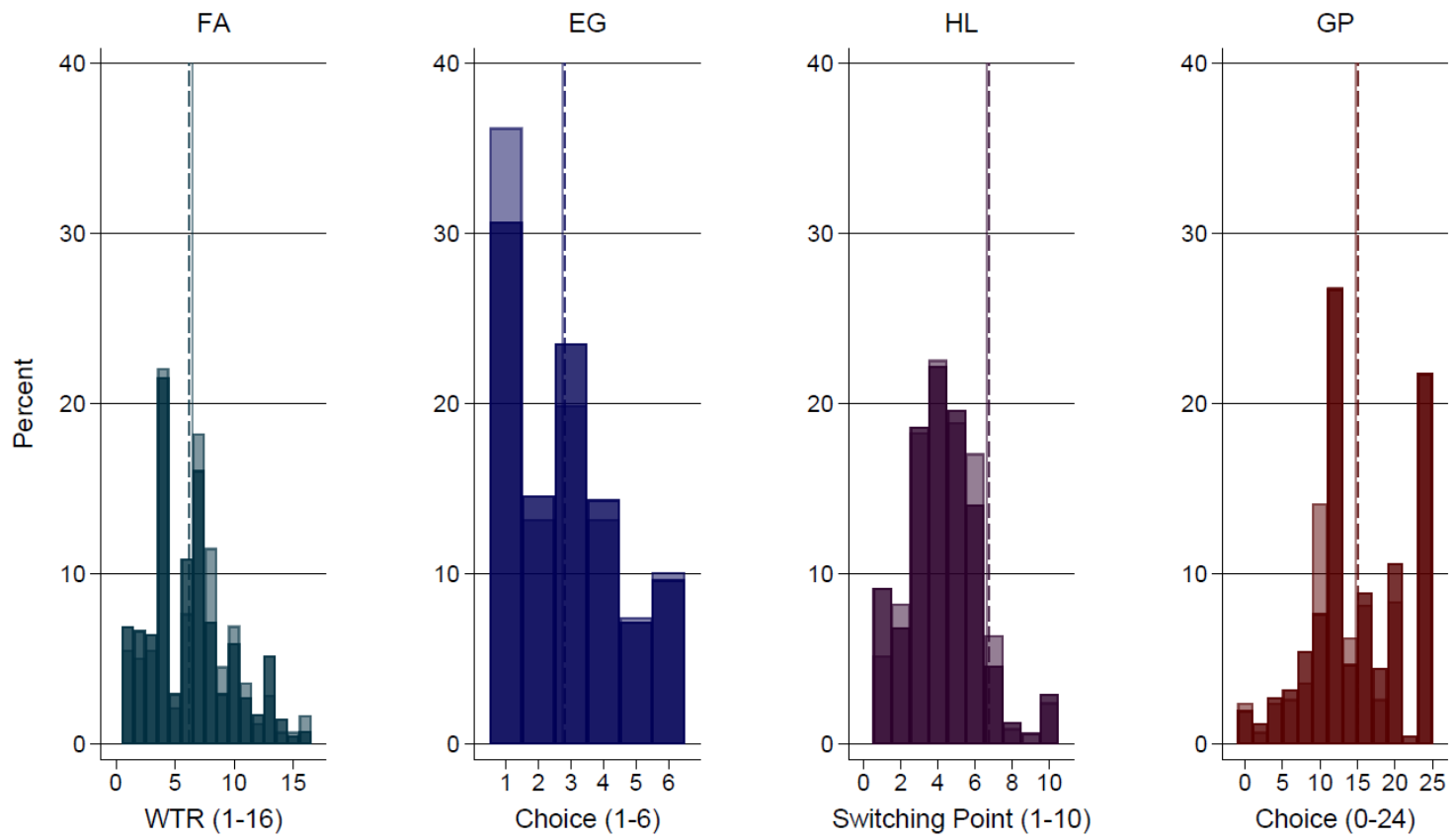

The light (dark) shaded bars represent the choices of subjects in the FLAT (INCENTIVES) condition.

Panel B: Professional Investors
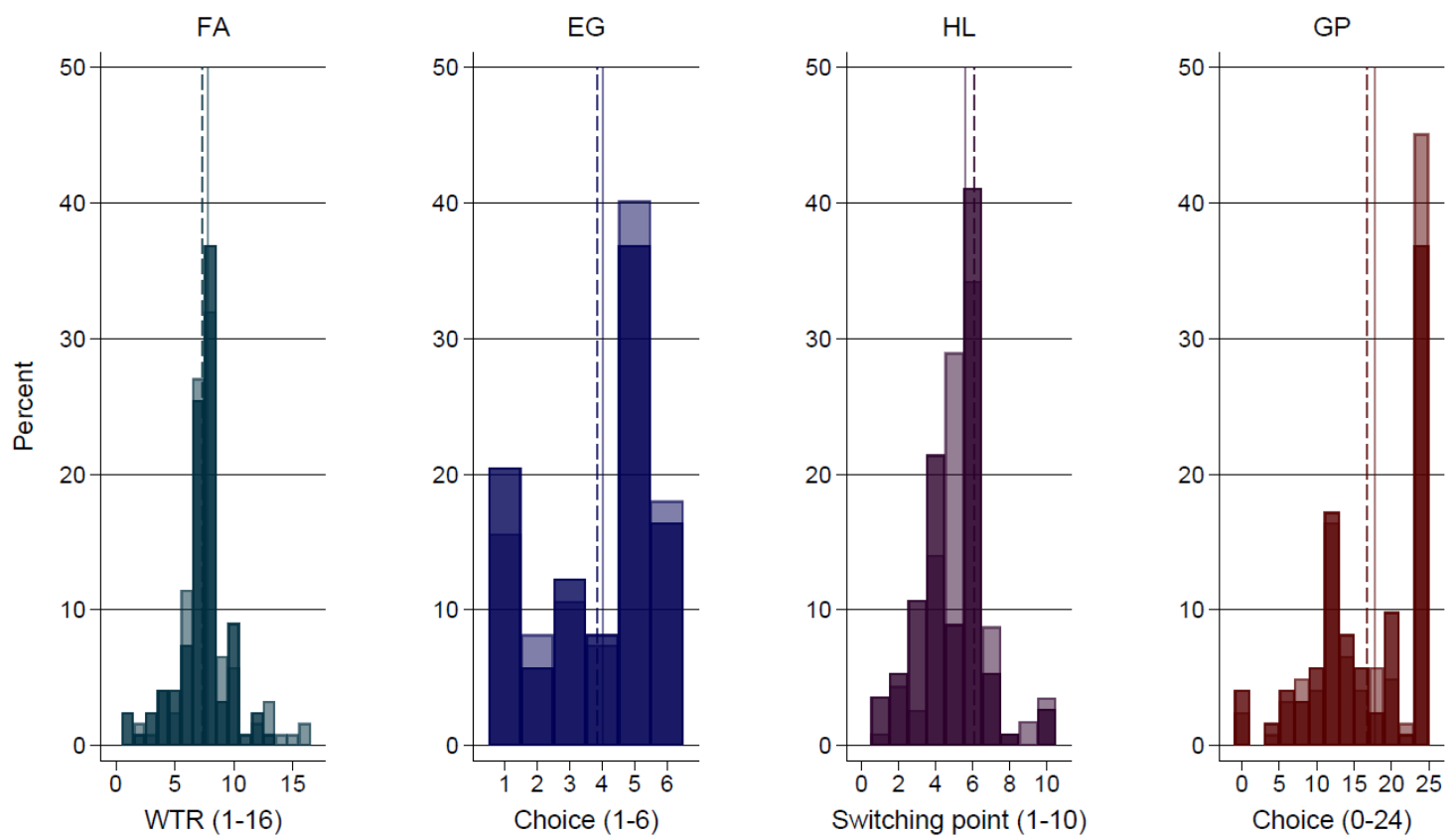

The light (dark) shaded bars represent the choices of subjects in the FLAT (INCENTIVES) condition. 
Figure A3 (continued): Effect of task-related incentives on risk-taking - Distributions

\section{Panel C: Students}
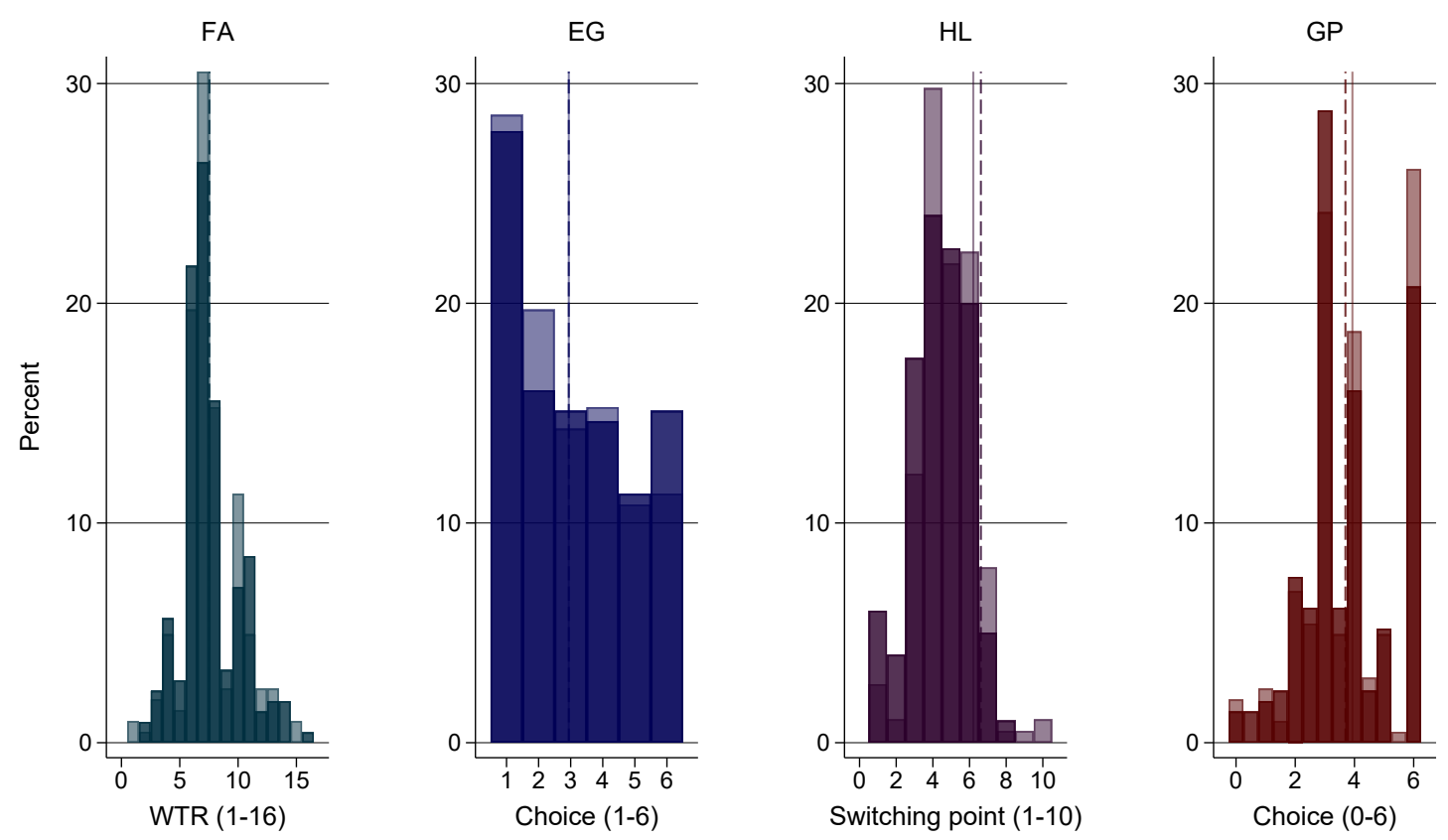

Notes: The figure shows the distributions of respondents' choices in the four experimental tasks (FA in emerald, EG in blue, HL in purple, and GP in maroon) by incentive condition, separately for each subject pool. The light (dark) shaded bars represent the choices of subjects in the FLAT (INCENTIVES) condition. The solid (dashed) line represents the mean in the FLAT (INCENTIVES) condition. In the FLAT condition, subjects receive a fixed participation reward only, amounting to $€ 12$ for private investors and professional investors, and to $€ 3$ for students. Subjects in the INCENTIVEs condition in addition are paid the earnings resulting from their choice in one randomly determined experimental task. Panel A represents choices in the private investor sample. Panel B (C) represents choices in the professional investor (student) sample. FA takes a value between 1 and 16, according to the certainty equivalent resulting from the last of the four choices in the staircase risk task. EG is the rank (1-6) of the gamble chosen from a menu of six 50/50 gambles, increasing in risk. HL is the number of decision rows left after switching to the higher-risk lottery, ranging from 0 to 10 . GP is the EUR amount invested in the risky project and takes values between 0 and 24 ( 0 and 6 for students). We report the p-values of Kolmogorov-Smirnov tests for equality of distributions in Table A2. 
Figure A4: Task-related incentives and extreme choices

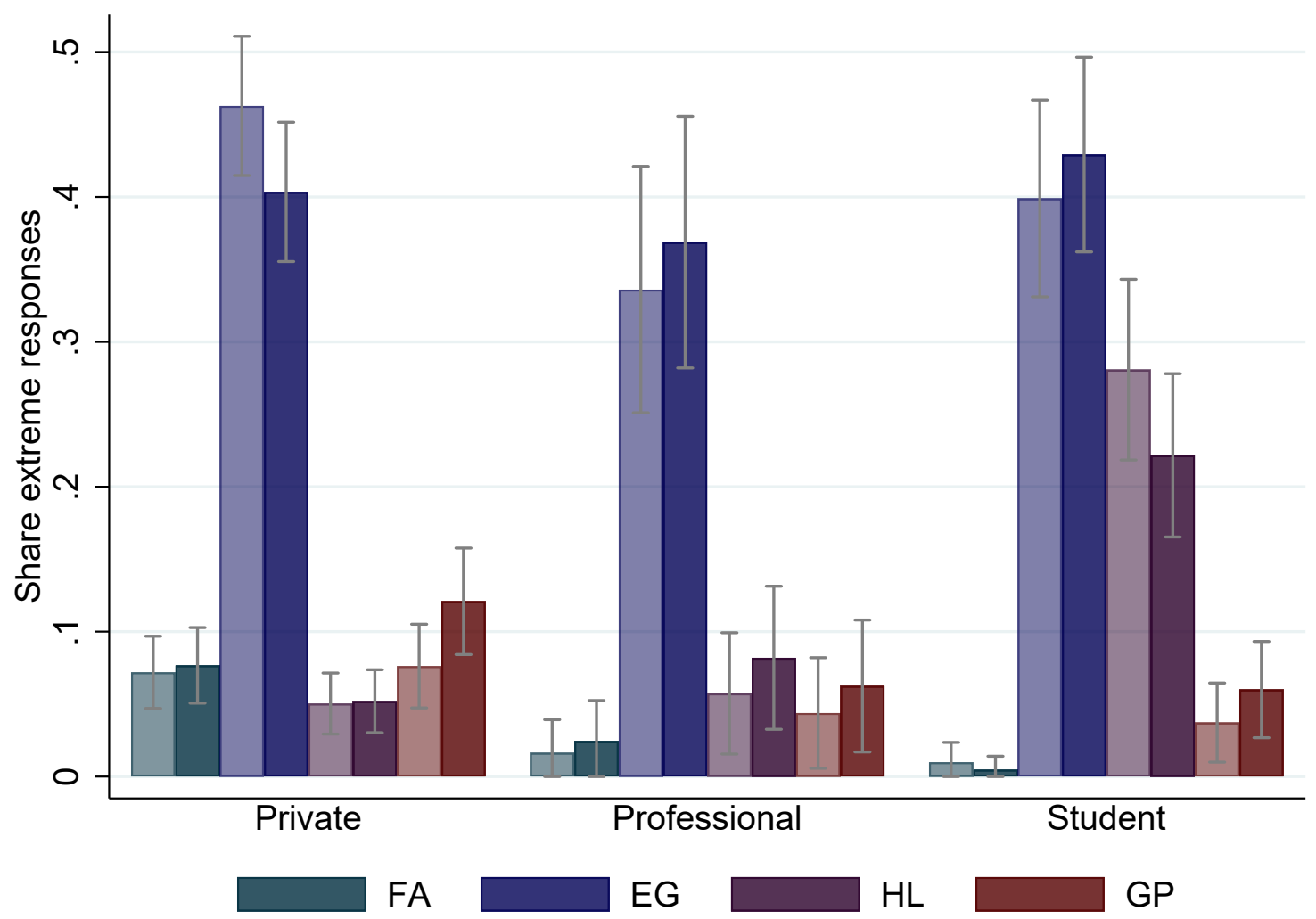

Notes: The figure shows the share of respondents who choose the least or the most risky option in the four experimental tasks (FA in emerald, EG in blue, HL in purple, and GP in maroon) by incentive condition, separately for the three subject pools. The light (dark) shaded bars represent the choices of subjects in the FLAT (INCENTIVES) condition. In the FLAT condition, subjects receive a fixed participation reward only, amounting to $€ 12$ for private investors and professional investors, and to $€ 3$ for students. Subjects in the INCENTIVES condition in addition are paid the earnings resulting from their choice in one randomly determined experimental task. The left block of bars represents probabilities of extreme choices in the private investor sample. The middle (right) block represents probabilities of extreme choices in the professional investor (student) sample. Error bars indicate 95\%-confidence intervals. We report the p-values of two-sided t-tests for equality of mean choices in Table A3. Note that the higher share of extreme answers for EG compared to the other tasks is not surprising as there are only 6 possible choices. Hence, the 2 extreme answers account for $1 / 3$ of the decision space. 
Figure A5: Consistency in responses across tasks - excluding HL

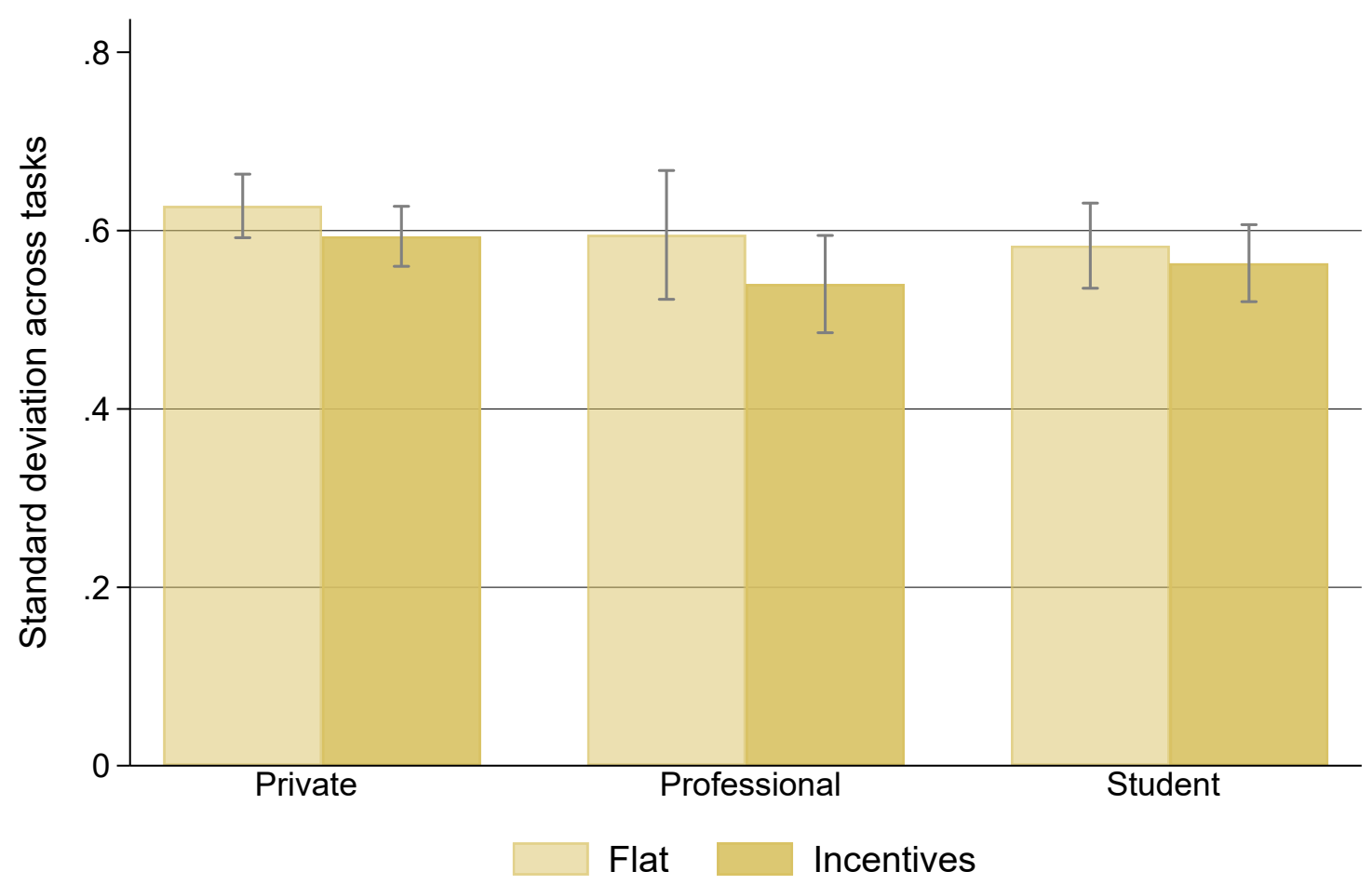

Notes: The figure compares the mean within-subject standard deviation for three of the four experimental tasks - excluding HL - by incentive condition separately for the three subject pools. We standardize choices in the single tasks by deducting the mean and dividing by the standard deviation of choices made in the given task in the relevant subject pool. We then calculate the within-subject standard deviation over a subject's three standardized choices. The light (dark) shaded bars refer to subjects in the FLAT (InCENTIVES) condition. In the FLAT condition, subjects receive a fixed participation reward only, amounting to $€ 12$ for private investors and professional investors, and to $€ 3$ for students. Subjects in the INCENTIVES condition in addition are paid the earnings resulting from their choice in one randomly determined experimental task. Error bars indicate 95\%-confidence intervals. 


\section{B Additional Tables}

Table A1: Descriptive statistics

Panel A. Private investors

\begin{tabular}{|c|c|c|c|c|c|}
\hline & $\overline{A L L}$ & INCENTIVES & $\begin{array}{l}\text { FLAT } \\
\end{array}$ & & \\
\hline & $\begin{array}{c}\text { Mean } \\
(\mathrm{SD})\end{array}$ & Mean & Mean & $\mathrm{P}$-value & Obs. \\
\hline & (1) & $(2)$ & (3) & (4) & $(5)$ \\
\hline Female & $\begin{array}{c}0.25 \\
(0.44)\end{array}$ & 0.28 & 0.23 & 0.102 & 809 \\
\hline Age & $\begin{array}{c}52.98 \\
(15.67)\end{array}$ & 53.53 & 52.44 & 0.323 & 809 \\
\hline HH net income & $\begin{array}{c}4,250 \\
(2,402)\end{array}$ & 4,151 & 4,347 & 0.288 & 676 \\
\hline Stock investor & $\begin{array}{c}0.79 \\
(0.41)\end{array}$ & 0.79 & 0.79 & 0.949 & 821 \\
\hline Smartphone & $\begin{array}{c}0.19 \\
(0.39)\end{array}$ & 0.18 & 0.19 & 0.750 & 821 \\
\hline Total time & $\begin{array}{c}16.33 \\
(10.07)\end{array}$ & 17.33 & 15.37 & 0.005 & 821 \\
\hline Payoff & $\begin{array}{c}21.71 \\
(16.38)\end{array}$ & 31.72 & 12.00 & 0.000 & 821 \\
\hline
\end{tabular}

Panel B. Professional investors

\begin{tabular}{|c|c|c|c|c|c|}
\hline & ALL & INCENTIVES & FLAT & \multirow[b]{2}{*}{$\mathrm{P}$-value } & \multirow[b]{2}{*}{ Obs } \\
\hline & $\begin{array}{c}\text { Mean } \\
(\mathrm{SD})\end{array}$ & Mean & $\overline{\text { Mean }}$ & & \\
\hline & (1) & (2) & (3) & (4) & (5) \\
\hline Female & $\begin{array}{c}0.11 \\
(0.31)\end{array}$ & 0.11 & 0.11 & 1.000 & 244 \\
\hline Age & $\begin{array}{l}41.89 \\
(9.50)\end{array}$ & 42.19 & 41.59 & 0.624 & 244 \\
\hline $\begin{array}{l}\text { Job position } \\
\text { - Fund manager } \\
\text { - Portfolio manager } \\
\text { - Analyst } \\
\text { - Risk manager }\end{array}$ & $\begin{array}{l}0.35 \\
0.18 \\
0.10 \\
0.07\end{array}$ & $\begin{array}{l}0.37 \\
0.15 \\
0.09 \\
0.08\end{array}$ & $\begin{array}{l}0.34 \\
0.20 \\
0.11 \\
0.06\end{array}$ & $\begin{array}{l}0.594 \\
0.241 \\
0.528 \\
0.453\end{array}$ & $\begin{array}{l}244 \\
244 \\
244 \\
244\end{array}$ \\
\hline Smartphone & $\begin{array}{c}0.05 \\
(0.22)\end{array}$ & 0.03 & 0.07 & 0.238 & 244 \\
\hline Total time & $\begin{array}{c}20.46 \\
(12.41)\end{array}$ & 22.77 & 18.15 & 0.003 & 244 \\
\hline Payoff & $\begin{array}{c}21.72 \\
(17.17)\end{array}$ & 31.43 & 12.00 & 0.000 & 244 \\
\hline
\end{tabular}

Table continues on next page. 
Table A1: cont.

Panel C. Students

\begin{tabular}{|c|c|c|c|c|c|c|}
\hline & ALL & INCENTIVES & FLAT & FLAT_HIGH & & \\
\hline & $\begin{array}{l}\text { Mean } \\
(\mathrm{SD})\end{array}$ & Mean & Mean & Mean & P-value & Obs. \\
\hline & (1) & $(2)$ & $(3)$ & (4) & (5) & $(6)$ \\
\hline Female & $\begin{array}{c}0.57 \\
(0.50)\end{array}$ & 0.58 & 0.54 & 0.59 & 0.584 & 628 \\
\hline Age & $\begin{array}{l}23.98 \\
(3.47)\end{array}$ & 24.16 & 23.71 & 24.06 & 0.352 & 628 \\
\hline Net income & $\begin{array}{c}749.02 \\
(385.16)\end{array}$ & 727.14 & 772.54 & 749.28 & 0.471 & 610 \\
\hline Stock investor & $\begin{array}{c}0.24 \\
(0.43)\end{array}$ & 0.23 & 0.26 & 0.23 & 0.668 & 628 \\
\hline Smartphone & $\begin{array}{c}0.04 \\
(0.19)\end{array}$ & 0.03 & 0.04 & 0.04 & 0.837 & 628 \\
\hline Total time & $\begin{array}{l}12.90 \\
(7.34)\end{array}$ & 14.35 & 12.52 & 11.81 & 0.001 & 628 \\
\hline Payoff & $\begin{array}{c}6.61 \\
(4.57)\end{array}$ & 7.67 & 3.00 & 9.00 & 0.000 & 628 \\
\hline
\end{tabular}

Notes: Panel A shows summary statistics for the 821 respondents in the private investor sample. Panel B shows summary statistics for the 244 respondents in the finance professionals sample. Panel C shows summary statistics for the 628 participants in the student sample. Information of respondents' household net income is only available for 676 of the 821 respondents in the private investor sample, due to non-response. Similarly, 18 of the 628 students choose "prefer not to answer". Note that private investor demographics have been elicited in an earlier survey wave and were not re-elicited for the purpose of our study. This causes demographics to be missing for a number of private investors who did not participate in this earlier survey. Stock investor is an indicator equal to one for participants who invest in stocks or stock mutual funds. Smartphone is an indicator of whether the respondent has participated in the experiment using a smartphone. Total time is the time (in minutes) a subject has spend to complete the entire experiment. Payoff is the final payoff participants receive after completing the experiment. It is fixed in the FLAT and FLAT_HIGH (students only) conditions. For subjects in the INCENTIVES condition, it depends on the choice and resulting outcome in one randomly determined experimental task. Task-related payoffs in the INCENTIVES condition are in addition to the fixed participation fee paid to subjects in the relevant FLAT condition. Potential payoffs in the student sample result from dividing payoff options presented to private investors and finance professionals by 4 . In Panels A (private investors) and B (finance professionals), column 4 reports p-values from a two-sided t-test of equal means between subjects in the INCENTIVES and FLAT condition. Given that there are three treatment conditions in the student sample, the p-value displayed in column 5 in Panel $\mathrm{C}$ refers to a F-test of whether when regressing each characteristic on the treatment group indicators, all coefficients are jointly zero. 
Table A2: Effect of task-related incentives on risk-taking

Panel A. Private investors

\begin{tabular}{lcccc}
\hline & FA & EG & HL & GP \\
\cline { 2 - 5 } & & & & \\
FLAT & 6.47 & 2.73 & 6.60 & 14.76 \\
INCENTIVES & 6.21 & 2.82 & 6.72 & 15.09 \\
$\Delta$ & -0.26 & 0.09 & 0.13 & 0.33 \\
t-test (p-value) & & & & \\
KS test (p-value) & 0.272 & 0.436 & 0.406 & 0.460 \\
Observations & 0.177 & 0.525 & 0.956 & 0.583 \\
\hline
\end{tabular}

Panel B. Professional investors

\begin{tabular}{lcccc}
\hline & FA & EG & HL & GP \\
\cline { 2 - 5 } & & & & \\
FLAT & 7.77 & 4.02 & 5.57 & 17.77 \\
INCENTIVES & 7.34 & 3.84 & 6.05 & 16.72 \\
$\Delta$ & -0.43 & -0.18 & $\mathbf{0 . 4 8}$ & -1.05 \\
t-test (p-value) & & & & \\
KS test (p-value) & 0.135 & 0.425 & 0.032 & 0.237 \\
Observations & 0.983 & 0.998 & 0.022 & 0.652 \\
\hline
\end{tabular}

Panel C. Students

\begin{tabular}{lcccc}
\hline & FA & EG & HL & GP \\
\cline { 2 - 5 } & & & & \\
FLATs & 7.55 & 3.11 & 6.62 & 3.70 \\
INCENTIVES & 7.55 & 2.94 & 6.21 & 3.93 \\
$\Delta$ & 0.00 & 0.17 & $\mathbf{0 . 4 1}$ & -0.24 \\
t-test (p-value) & & & & \\
KS test (p-value) & 0.984 & 0.333 & 0.008 & 0.116 \\
& 0.996 & 0.983 & 0.125 & 0.345 \\
Observations & & & & \\
\hline
\end{tabular}

Notes: The table reports differences in risk-taking in the four different experimental task by incentive condition and subject pool. In the FLAT condition, subjects receive a fixed participation reward only, amounting to $€ 12$ for private investors and professional investors, and to $€ 3$ for students. Subjects in the INCENTIVES condition in addition are paid the earnings resulting from their choice in one randomly determined experimental task. Panels A, B and C show differences by incentive condition for the 821 subjects in the private investor sample, the 244 subjects in the professional investor sample, and the 638 respondents in the student sample, respectively. Mean differences that are significant at least at the 5 percent level are printed in bold. We report p-values of a two sided t-test of equal means and p-values of a Kolmogorov-Smirnov test of equal distributions. 
Table A3: Probability of extreme choices

\begin{tabular}{|c|c|c|c|c|c|c|c|c|c|c|}
\hline & \multirow{2}{*}{$\begin{array}{c}\text { All } \\
(1)\end{array}$} & \multicolumn{3}{|c|}{ Private investors } & \multicolumn{3}{|c|}{ Professional investors } & \multicolumn{3}{|c|}{ Students } \\
\hline & & $\begin{array}{l}\text { INCENTIVES } \\
(2)\end{array}$ & $\begin{array}{c}\text { FLAT } \\
(3)\end{array}$ & $\begin{array}{c}\text { P-value } \\
(4)\end{array}$ & $\begin{array}{c}\text { INCENTIVES } \\
(5)\end{array}$ & $\begin{array}{l}\text { FLAT } \\
(6)\end{array}$ & $\begin{array}{c}\text { P-value } \\
(7)\end{array}$ & $\begin{array}{l}\text { INCENTIVES } \\
(8)\end{array}$ & $\begin{array}{l}\text { FLAT } \\
(9)\end{array}$ & $\begin{array}{c}\text { P-value } \\
(10)\end{array}$ \\
\hline \multicolumn{11}{|c|}{ Extreme choice } \\
\hline FA & 0.04 & 0.08 & 0.07 & $\begin{array}{l}-0.00 \\
(0.79)\end{array}$ & 0.02 & 0.02 & $\begin{array}{l}-0.01 \\
(0.65)\end{array}$ & 0.00 & 0.01 & $\begin{array}{c}0.01 \\
(0.54)\end{array}$ \\
\hline EG & 0.41 & 0.40 & 0.46 & $\begin{array}{c}0.06 \\
(0.09)\end{array}$ & 0.37 & 0.34 & $\begin{array}{l}-0.03 \\
(0.59)\end{array}$ & 0.43 & 0.40 & $\begin{array}{l}-0.03 \\
(0.53)\end{array}$ \\
\hline HL & 0.07 & 0.12 & 0.08 & $\begin{array}{l}-0.04 \\
(0.06)\end{array}$ & 0.06 & 0.04 & $\begin{array}{l}-0.02 \\
(0.53)\end{array}$ & 0.06 & 0.04 & $\begin{array}{l}-0.02 \\
(0.30)\end{array}$ \\
\hline GP & 0.12 & 0.05 & 0.05 & $\begin{array}{l}-0.00 \\
(0.92)\end{array}$ & 0.08 & 0.06 & $\begin{array}{l}-0.02 \\
(0.45)\end{array}$ & 0.22 & 0.28 & $\begin{array}{c}0.06 \\
(0.17)\end{array}$ \\
\hline Observations & 1,693 & 404 & 417 & 821 & 122 & 122 & 244 & 212 & 203 & 415 \\
\hline
\end{tabular}

Notes: The table reports differences in the propensity to choose the least or the most risky option in the four different tasks, by incentive condition and subject pool. In the FLAT condition, subjects receive a fixed participation reward only, amounting to $€ 12$ for private investors and professional investors, and to $€ 3$ for students. Subjects in the INCENTIVES condition in addition are paid the earnings resulting from their choice in one randomly determined experimental task. We report p-values of a two sided t-test of equal means for each sample. 
Table A4: Effort and consistency of choices across tasks

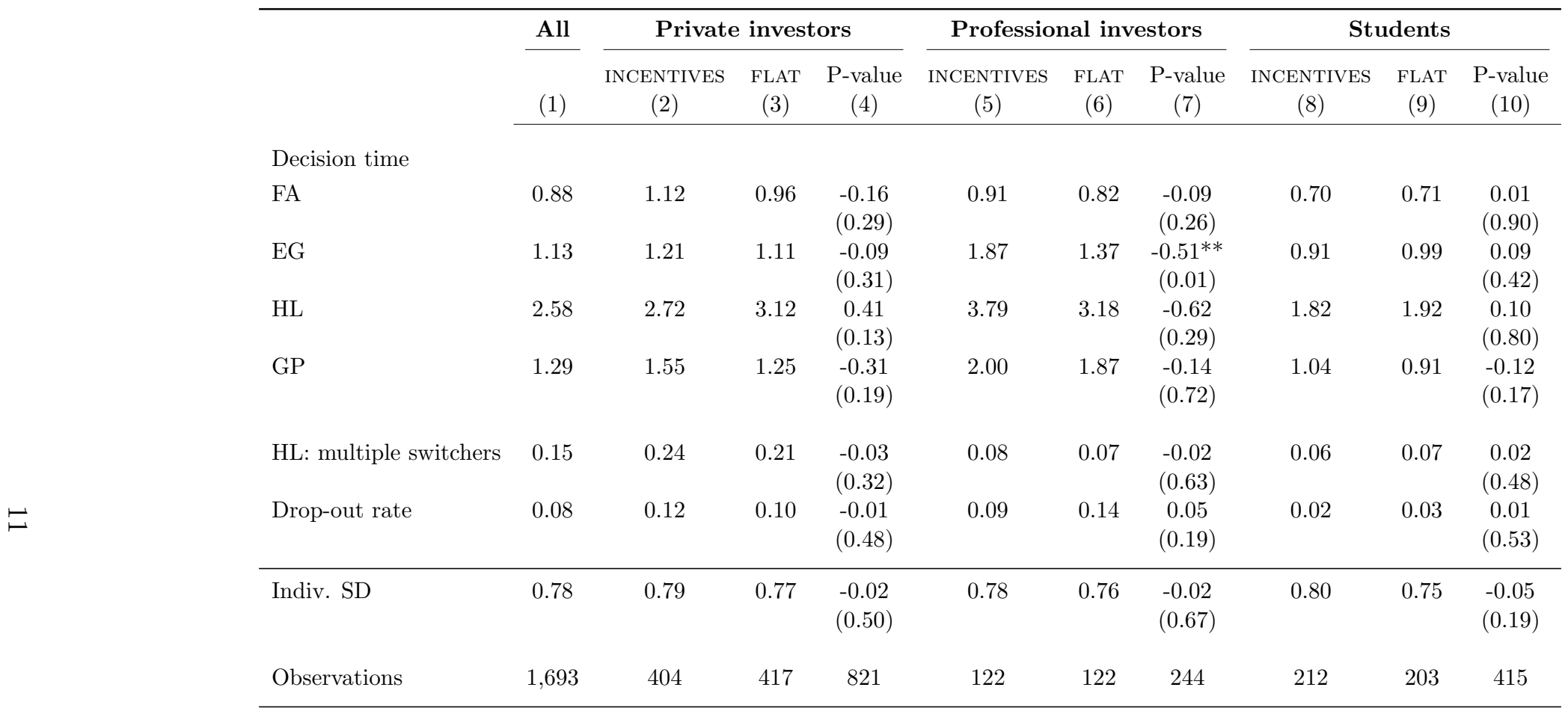

Notes: The table reports differences in task-specific decision times, frequencies of multiple switching in the HL task, drop-out rates as well as the within-subject standard deviation of an individual's choices across the four tasks by incentive condition and subject pool. In the FLAT condition, subjects receive a fixed participation reward only, amounting to $€ 12$ for private investors and professional investors, and to $€ 3$ for students. Subjects in the INCENTIVES condition in addition are paid the earnings resulting from their choice in one randomly determined experimental task. Drop out rates are calculated based on the overall number of respondents who started the experiment $(\mathrm{N}=1,882)$, of which 1,727 completed it. To calculate the within-subject standard deviation, we standardize subjects' choices in the four experimental tasks by subtracting the mean and dividing by the standard deviation of the distribution of choices in the respective task in the relevant subject pool. For each subject, we then calculate the standard deviation over the standardized choices in the four experimental tasks. We report p-values of two sided t-tests of equal means. 


\section{Additional Analyses}

\section{Details on the power analysis}

Our goal is to provide the basis for conclusive inference, including the case where we would not reject the null hypothesis of no effect of monetary incentivization. To this end, we seek to achieve a sufficiently high power for our statistical tests. In the absence of an indication of how large the effect of incentivization on average choices might be, we followed the reference points suggested by Cohen (1988) for behavioral sciences. As a lower bound for our analysis, we sought a probability of $90 \%$ to detect a 'small' effect size of less than 0.5 of a standard deviation. ${ }^{4}$ This result corresponds to a sample size of at least $N=85$ per incentive condition. For the most difficult to recruit sample, that of professional investors, we targeted a sample size of $N=100$. For the samples of students and private investors, which are easier to recruit, we aimed at sample sizes that would allow us to detect even smaller effect sizes of $d=0.33$ and $d=0.20$ (the latter being the lowest threshold for power analysis as suggested by Cohen (1988)), respectively, rounding to targeted sample sizes of $N=200$ and $N=500$.

Table A5: Power analysis

\begin{tabular}{l}
\hline \\
\cline { 2 - 3 } $\begin{array}{l}\text { Private investors } \\
\left(N_{1}=404, N_{2}=417\right)\end{array}$ \\
$\begin{array}{l}\text { Professional investors } \\
\left(N_{1}=122, N_{2}=122\right)\end{array}$
\end{tabular}

Our average realized sample sizes per group were 410, 122, and 209 for private investors, professional investors, and students, respectively. These numbers are all well above the lower

\footnotetext{
${ }^{4}$ Cohen (1988) argues that a 'medium' effect size of $d=0.5$ is 'large enough to be visible to the naked eye'. To put effect sizes into the perspective of risk elicitation experiments, we refer to the extensive meta-study by Filippin and Crosetto (2016), who analyze the effect of gender on risk-taking. They find an average effect size of $d=0.55$ for both the investment game and the gamble-choice task, and $d=0.17$ for the multiple price list. However, while, some controversy persists on the effect of gender on risk-taking, the importance of incentivizing preference elicitation tasks seems to be almost universally accepted among experimental economists. We therefore believe that a threshold below the median effect of gender is a conservative benchmark for the presumed effect of incentivization.
} 
bound of $N=85$ to detect effect sizes below $d=0.5$ with $90 \%$ power. The corresponding ex ante effect sizes we are able to detect given our realized numbers of observations are summarized in Table A5. 


\section{Instructions of the Online Experiment}

\section{Intro screen incentives treatment}

universität GOETHE

universitä

Thank you for participating in this study!

The survey takes about 15-20 minutes. Participation is voluntary and anonymous. Responses are analyzed for the purpose of academic research only.

We reward your participation with real monetary benefits. The starting value of your reward is 12 EUR. Depending on your answers in the survey, your payoff can increase or decrease. On average, you can expect a payoff of 36 EUR. You can choose whether to receive your final payoff via bank transfer or as an online Amazon gift card.

In the first six tasks of this survey, we ask you to choose among several options with different payoff profiles. To determine your final payoff, at the end of the survey, one of these six tasks is selected at random. The outcome of one of your decisions in the respective task determines by how much your final payoff increases above or decreases below the initial 12 EUR. All tasks are equally likely to be relevant for your payoff.

Note that there are no "right" or "wrong" answers to the questions in this survey. This questionnaire is about your own preferences. Always choose the option that you prefer.

Note:

The survey includes content that is not displayed optimally on smartphones. We therefore ask you to answer the survey on a desktop computer, laptop or tablet, if possible.

Amazon gift cards are distributed via e-mail. If you choose to receive a gift card, please indicate your e-mail address at the end of the survey. If you prefer to receive your payoff via bank transfer, please indicate your bank details at the end of the survey. E-mail addresses and bank details are stored separately from your answers and are deleted immediately after sending out the rewards.

If you have any questions, you can contact us by e-mail (finanzforum@finance.uni-frankfurt.de). 


\section{Intro screen flat treatment}

universität

Thank you for participating in this study!

The survey takes about 15-20 minutes. Participation is voluntary and anonymous. Responses are analyzed for the purpose of academic research only

We reward your participation with real monetary benefits. The value of your reward is 12 EUR. You can choose whether to receive your final payoff via bank transfer or as an online Amazon gift card.

In the first six tasks of this survey, we ask you to choose among several options with different hypothetical payoff profiles. Note that the payoff amounts that result from your decisions will not actually be paid out

Note that there are no "right" or "wrong" answers to the questions in this survey. This questionnaire is about your own preferences Always choose the option that you prefer.

\section{Note:}

The survey includes content that is not displayed optimally on smartphones. We therefore ask you to answer the survey on a desktop computer, laptop or tablet, if possible.

Amazon gift cards are distributed via e-mail. If you choose to receive a gift card, please indicate your e-mail address at the end of the survey. If you prefer to receive your payoff via bank transfer, please indicate your bank details at the end of the survey. E-mail addresses and bank details are stored separately from your answers and are deleted immediately after sending out the rewards.

If you have any questions, you can contact us by e-mail (finanzforum@finance.uni-frankfurt.de). 
The staircase procedure by Falk et al. $(2016,2018)$ (FA)

\section{Task: Decision sequence}

Instructions

In the following four decisions, we ask you to choose between receiving a fixed amount (Option A) or participating in a lottery (Option B) which either pays 45 EUR or 0 EUR with equal probability (50/50).

The payoffs of the lottery are the same in each of the four decisions. The fixed amount is different in each decision.

If one of these four decisions is drawn at random to determine your payoff your final earnings result as follows. If you choose to participate in the lottery, a fair coin is tossed to determine the outcome of the lottery. If the coin comes up "heads", your payoff increases by the positive amount, in addition to the initial payment. If the coin comes up "tails", 
Task: Decision sequence

Decision 1 of 4

Which option do you prefer?

Option A: You receive a fixed amount of 24 EUR.

Option B: You receive either 0 EUR or 45 EUR with equal probability (50/50).

Option A: You receive a fixed amount of 24 EUR.

Option B: You receive either O EUR or 45 EUR with equal probability (50/50). 
Task: Decision sequence

Decision 2 of 4

Which option do you prefer?

Option A: You receive a fixed amount of 36 EUR.

Option B: You receive either 0 EUR or 45 EUR with equal probability (50/50). 
Task: Decision sequence

Decision 3 of 4

Which option do you prefer?

Option A: You receive a fixed amount of 42 EUR.

Option B: You receive either 0 EUR or 45 EUR with equal probability (50/50). 


\begin{tabular}{|c|c|c|c|}
\hline $\begin{array}{l}\text { universität } \\
\text { innsbruck }\end{array}$ & $\frac{\text { GOETHE }}{\text { UNIVERSTAT }}$ & Resume later & Exit and clear survey \\
\hline
\end{tabular}

Task: Decision sequence

Decision 4 of 4

Which option do you prefer?

Option A: You receive a fixed amount of 45 EUR.

Option B: You receive either 0 EUR or 45 EUR with equal probability (50/50). 
The gamble-choice task by Eckel and Grossman (2002) (EG)

\section{Task: Coin toss}

You are asked to choose your favorite option among the six options displayed in the table below.

Each option has two possible payoffs. A coin is tossed to determine which of the two amounts is paid out. Both payoffs hence result with equal probability.

$\begin{array}{ccc}\text { Option } & \text { Coin comes up "heads" } & \text { Coin comes up "tails" } \\ 1 & \text { (payoff in EUR) } & \text { (payoff in EUR) } \\ 2 & 21 \text { EUR } & 21 \text { EUR } \\ 3 & 27 \text { EUR } & 18 \text { EUR } \\ 4 & 33 \text { EUR } & 15 \text { EUR } \\ 5 & 39 \text { EUR } & 12 \text { EUR } \\ 6 & 45 \text { EUR } & 9 \text { EUR } \\ & 52 \text { EUR } & 2 \text { EUR }\end{array}$

Please choose your preferred option.

If this decision is drawn at random to determine your payoff, your final earnings result as follows. To determine which of two amounts is paid out, a fair coin is tossed. If the coin comes up "heads", your payoff increases by the higher amount, in addition to the initial payment. If the coin comes up "tails", your payoff increases by the lower amount, in addition to the initial payment.

Option 1 [Heads: 21 EUR or Tails: 21 EUR]

Option 2 [Heads: 27 EUR or Tails: 18 EUR]

Option 3 [Heads: 33 EUR or Tails: 15 EUR]

Option 4 [Heads: 39 EUR or Tails: 12 EUR]

Option 5 [Heads: 45 EUR or Tails: 9 EUR]

Option 6 [Heads: 52 EUR or Tails: 2 EUR] 
The paired lottery choice task by Holt and Laury (2002) (HL)

universität

GOETHE

Task: Probability scenarios

You are asked to choose between two options, Option A and Option B, in each of the 10 scenarios below.

- Option A is a lottery that pays either 24.00 EUR or 19.20 EUR.

- Option B is a lottery that pays either 46.20 EUR or 1.20 EUR.

Starting from scenario 1 , the chances of the higher payoff increase in each subsequent scenario as you move down the table.

\begin{tabular}{|c|c|c|c|c|c|c|c|c|c|}
\hline II & & \multirow{2}{*}{\multicolumn{2}{|c|}{ Opption A }} & & II & \multirow{2}{*}{\multicolumn{4}{|c|}{ Option B }} \\
\hline ॥ & & & & & $\|$ & & & & \\
\hline II & & \multicolumn{2}{|c|}{ or: } & & ॥ & \multicolumn{4}{|c|}{ or: } \\
\hline \multicolumn{2}{|c|}{ || Probability } & Payoff & Probability & Payoff & ॥ & Probability & Payoff & | Probability & Payoff \\
\hline (1) II & $10 \%$ & 24.00 EUR & $90 \%$ & 19.20 EUR & II & $10 \%$ & 46.20 EUR & $90 \%$ & 1.20 EUR \\
\hline (2) II & $20 \%$ & 24.00 EUR & $80 \%$ & 19.20 EUR & ॥ & $20 \%$ & 46.20 EUR & $80 \%$ & 1.20 EUR \\
\hline (3) II & $30 \%$ & 24.00 EUR & $70 \%$ & 19.20 EUR & II & $30 \%$ & 46.20 EUR & $70 \%$ & 1.20 EUR \\
\hline (4) II & $40 \%$ & 24.00 EUR & $60 \%$ & 19.20 EUR & II & $40 \%$ & 46.20 EUR & $60 \%$ & 1.20 EUR \\
\hline (5) ॥ & $50 \%$ & 24.00 EUR & $50 \%$ & 19.20 EUR & II & $50 \%$ & 46.20 EUR & $50 \%$ & 1.20 EUR \\
\hline (6) II & $60 \%$ & 24.00 EUR & $40 \%$ & 19.20 EUR & II & $60 \%$ & 46.20 EUR & $40 \%$ & 1.20 EUR \\
\hline (7) II & $70 \%$ & 24.00 EUR & $30 \%$ & 19.20 EUR & II & $70 \%$ & 46.20 EUR & $30 \%$ & 1.20 EUR \\
\hline (8) II & $80 \%$ & 24.00 EUR & $20 \%$ & 19.20 EUR & . & $80 \%$ & 46.20 EUR & $20 \%$ & 1.20 EUR \\
\hline (9) II & $90 \%$ & 24.00 EUR & $10 \%$ & 19.20 EUR & $\|$ & $90 \%$ & 46.20 EUR & $10 \%$ & 1.20 EUR \\
\hline (10) II & $100 \%$ & 24.00 EUR & $0 \%$ & 19.20 EUR & $\|$ & $100 \%$ & 46.20 EUR & $0 \%$ & 1.20 EUR \\
\hline \| & & & & & & & & & \\
\hline
\end{tabular}

Please indicate whether you prefer Option A or Option B in each of the 10 scenarios.

If this decision is drawn at random to determine your payoff, your final earnings result as follows. In a first step, one of the 10 scenarios is selected at random. To determine outcome of the option you selected for this scenario, one ball is drawn at random from an urn containing 100 blue and red balls. The proportion of blue and red balls corresponds to the probabilities of respective scenario (e.g., 10 blue and 90 red balls for Scenario 1). If a blue (red) ball is drawn, your payoff fincreases by the higher (lower) amount, in addition to the initial payment.

\begin{tabular}{|c|c|c|c|}
\hline & Option A & Option B & \\
\hline $\begin{array}{l}\text { 24.00 EUR with } 10 \% \text { probability or } \\
19.20 \text { EUR with } 90 \% \text { probability }\end{array}$ & & & $\begin{array}{l}\text { 46.20 EUR with } 10 \% \text { probability or } \\
1.20 \text { EUR with } 90 \% \text { probability }\end{array}$ \\
\hline $\begin{array}{l}24.00 \text { EUR with } 20 \% \text { probability or } \\
19.20 \text { EUR with } 80 \% \text { probability }\end{array}$ & & & $\begin{array}{l}46.20 \text { EUR with } 20 \% \text { probability or } \\
1.20 \text { EUR with } 80 \% \text { probability }\end{array}$ \\
\hline $\begin{array}{l}24.00 \text { EUR with } 30 \% \text { probability or } \\
19.20 \text { EUR with } 70 \% \text { probability }\end{array}$ & & & $\begin{array}{l}46.20 \text { EUR with } 30 \% \text { probability or } \\
1.20 \text { EUR with } 70 \% \text { probability }\end{array}$ \\
\hline $\begin{array}{l}24.00 \text { EUR with } 40 \% \text { probability or } \\
19.20 \text { EUR with } 60 \% \text { probability }\end{array}$ & & & $\begin{array}{l}46.20 \text { EUR with } 40 \% \text { probability or } \\
1.20 \text { EUR with } 60 \% \text { probability }\end{array}$ \\
\hline $\begin{array}{l}24.00 \text { EUR with } 50 \% \text { probability or } \\
19.20 \text { EUR with } 50 \% \text { probability }\end{array}$ & & & $\begin{array}{l}46.20 \text { EUR with } 50 \% \text { probability or } \\
1.20 \text { EUR with } 50 \% \text { probability }\end{array}$ \\
\hline $\begin{array}{l}24.00 \text { EUR with } 60 \% \text { probability or } \\
19.20 \text { EUR with } 40 \% \text { probability }\end{array}$ & & & $\begin{array}{l}46.20 \text { EUR with } 60 \% \text { probability or } \\
1.20 \text { EUR with } 40 \% \text { probability }\end{array}$ \\
\hline $\begin{array}{l}24.00 \text { EUR with } 70 \% \text { probability or } \\
19.20 \text { EUR with } 30 \% \text { probability }\end{array}$ & & & $\begin{array}{l}46.20 \text { EUR with } 70 \% \text { probability or } \\
1.20 \text { EUR with } 30 \% \text { probability }\end{array}$ \\
\hline $\begin{array}{l}24.00 \text { EUR with } 80 \% \text { probability or } \\
19.20 \text { EUR with } 20 \% \text { probability }\end{array}$ & & & $\begin{array}{l}46.20 \text { EUR with } 80 \% \text { probability or } \\
1.20 \text { EUR with } 20 \% \text { probability }\end{array}$ \\
\hline $\begin{array}{l}24.00 \text { EUR with } 90 \% \text { probability or } \\
19.20 \text { EUR with } 10 \% \text { probability }\end{array}$ & & & $\begin{array}{l}46.20 \text { EUR with } 90 \% \text { probability or } \\
1.20 \text { EUR with } 10 \% \text { probability }\end{array}$ \\
\hline 24.00 EUR with $100 \%$ probability & & & 46.20 EUR with $100 \%$ probability \\
\hline
\end{tabular}




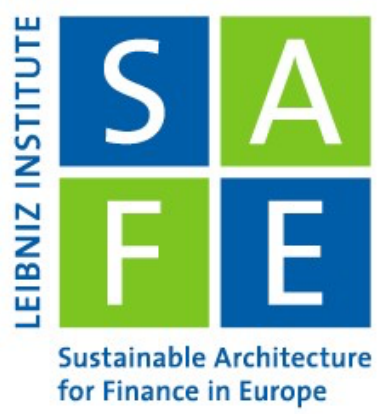

\section{Recent Issues}

No. 285 Elena Carletti, Tommaso Oliviero, Marco Pagano, Loriana Pelizzon, Marti G. Subrahmanyam

No. 284 Monica Billio, Michele Costola, Iva Hristova, Carmelo Latino, Loriana Pelizzon

No. 283 Jannis Bischof, Christian Laux, Christian Leuz

No. 282 Daniel Munevar, Grygoriy Pustovit

No. 281 Kevin Bauer

No. 280 Konstantin Bräuer, Andreas Hackethal, Tobin Hanspal

No. 279 Tobin Hanspal, Annika Weber, Johannes Wohlfart

No. 278 Sandra Eckert

No. 277 Dominique M. Lammer, Tobin Hanspal, Andreas Hackethal

No. 276 Massimiliano Caporin, Loriana Pelizzon, Alberto Plazzi

No. 275 Loriana Pelizzon, Max Riedel, Zorka Simon, Marti Subrahmanyam

No. 274 Christopher Busch, Alexander Ludwig

No. 273 Di Bu, Tobin Hanspal, Yin Liao, Yong Liu he COVID-19 Shock and Equity Shortfall:

Firm-Level Evidence from Italy

Inside the ESG Ratings: (Dis)agreement and Performance

Accounting for Financial Stability: Bank Disclosure and Loss Recognition in the Financial Crisis

Back to the Future: A Sovereign Debt Standstill Mechanism IMF Article VIII, Section 2 (b)

How did we do? The Impact of Relative Performance Feedback on Intergroup Hostilities

Consuming Dividends

Exposure to the COVID-19 Stock Market Crash and its Effect on Household Expectations

EU Agencies in Banking and Energy Between Institutional and Policy Centralisation

Who Are the Bitcoin Investors? Evidence from Indirect Cryptocurrency Investments

Does Monetary Policy Impact International Market Co-Movements?

Collateral Eligibility of Corporate Debt in the Eurosystem

Higher-Order Income Risk Over the Business Cycle

Financial Literacy and Self-Control in FinTech: Evidence from a Field Experiment on Online Consumer Borrowing 